\title{
Systematic study of homogenization and the utility of circular simplified representative volume element
}

Mathematics and Mechanics of Solids 2019, Vol. 24(9) 296I-2985 (C) The Author(s) 2019 Article reuse guidelines: sagepub.com/journals-permissions DOI: $10.1177 / 1081286518823834$ journals.sagepub.com/home/mms (SAGE

\author{
Soheil Firooz \\ Department of Mechanical Engineering, Bilkent University, Ankara, Turkey
}

\author{
Saba Saeb \\ Department of Applied Mechanics, University of Erlangen-Nuremberg, Erlangen, Germany
}

George Chatzigeorgiou
LEM3-UMR 7239 CNRS, Arts et Metiers ParisTech Metz, Metz, France

Fodil Meraghni

LEM3-UMR 7239 CNRS, Arts et Metiers ParisTech Metz, Metz, France

\section{Paul Steinmann}

Department of Applied Mechanics, University of Erlangen-Nuremberg, Erlangen, Germany;

School of Engineering, University of Glasgow, Glasgow, UK

\author{
Ali Javili \\ Department of Mechanical Engineering, Bilkent University, Ankara, Turkey
}

Received 7 October 2018; accepted I5 December 2018

\begin{abstract}
Although both computational and analytical homogenization are well-established today, a thorough and systematic study to compare them is missing in the literature. This manuscript aims to provide an exhaustive comparison of numerical computations and analytical estimates, such as Voigt, Reuss, Hashin-Shtrikman, and composite cylinder assemblage. The numerical computations are associated with canonical boundary conditions imposed on either tetragonal, hexagonal, or circular representative volume elements using the finite-element method. The circular representative volume element is employed to capture an effective isotropic material response suitable for comparison with associated analytical estimates. The analytical results from composite cylinder assemblage are in excellent agreement with the numerical results obtained from a circular representative volume element. We observe that the circular representative volume element renders identical responses for both linear displacement and periodic boundary conditions. In addition, the behaviors of periodic and random microstructures with different inclusion distributions are examined under various boundary conditions. Strikingly, for some specific microstructures, the effective shear modulus does not lie within the Hashin-Shtrikman
\end{abstract}

Corresponding author:

Ali Javili, Department of Mechanical Engineering, Bilkent University, 06800 Ankara, Turkey.

Email: ajavili@bilkent.edu.tr 
bounds. Finally, numerical simulations are carried out at finite deformations to compare different representative volume element types in the nonlinear regime. Unlike other canonical boundary conditions, the uniform traction boundary conditions result in nearly identical effective responses for all types of representative volume element, indicating that they are less sensitive with respect to the underlying microstructure. The numerical examples furnish adequate information to serve as benchmarks.

\section{Keywords}

Homogenization, hexagonal representative volume element, circular representative volume element, Hashin-Shtrikman bounds, composite cylinder assemblage, composites

\section{Introduction}

Heterogeneous materials possess more complex behavior than their associated constituents. Therefore, composites have been the subject of increasing interest in many engineering applications in past decades. The mechanical behavior of heterogeneous materials is highly dependent on their microstructural characteristics. Conducting experiments on numerous materials with various phases is not practical. Also, performing a numerical simulation on the whole macrostructure would involve using a huge number of variables, which is extremely complicated, if not impossible. As a result, a micromechanically based determination of the overall response of composites is of great significance.

Multiscale methods have been developed to determine the overall response of heterogeneous media in terms of the constitutive behavior of their underlying microstructures. Multiscale methods are categorized into concurrent methods and homogenization methods. In concurrent methods [1-4], the problems at the microscopic and macroscopic scales are solved simultaneously, requiring a strong coupling between the two scales. In the homogenization method [5-15], the microproblem and macroproblem are solved separately. Homogenization, pioneered by Hill [16, 17] and Ogden [18], relies on (i) the assumption of the separation of the length scale between the micro- and macroproblem and (ii) the energy equivalence between the two scales, known as the Hill-Mandel condition [16, 19]. Furthermore, homogenization methods fall into analytical homogenization and computational homogenization. Pioneering contributions in analytical homogenization include those of Voigt [20], Reuss [21], Eshelby [22], Hashin and colleagues [23, 24], Hill [25, 26], Walpole [27], Mori and Tanaka [28], and Willis [29]; the theory was later extended [30-38]. See Klusemann et al. [39] for some comparisons of analytical and computational approaches of micromechanics. Despite providing useful information, the analytical homogenization approach requires certain simplifications to the microstructure, such as its geometry and distribution pattern. By contrast, the computational homogenization method is capable of dealing with such complexities; thus, it has been widely adopted in the past decades [40-55]. For detailed reviews on computational homogenization, see Saeb et al. [56], Geers et al. [57], and Charalambakis et al. [58]. Computational homogenization essentially involves calculating macroscopic quantities from the solution of a boundary value problem at the microscale. Recently, a number of methods have been developed to reduce the computational cost and increase the accuracy of multiscale analysis [59-63].

The average field theory $[64,65]$ is employed to bridge microscopic quantities to their macroscopic counterparts. This theory relates the properties of continua at the macroscale to the volume averages of their counterparts at the microscale. In doing so, the boundary conditions at the microscale are chosen such that the Hill-Mandel condition is satisfied. A broader group of admissible boundary conditions to fill the gap between the homogeneous boundary conditions was derived [66]. The computational implementation algorithms of displacement boundary conditions (DBCs) and periodic boundary conditions (PBCs) are discussed by Yuan and Fish [67] and Nguyen et al. [68]. The effects of various boundary conditions on the overall behavior of periodic unidirectional linear composites has been investigated [69]. Furthermore, a mixed-type boundary condition composed of linear DBCs and constant traction boundary conditions (TBCs) has been proposed [70-72] to obtain an elasticity tensor that lies between the elasticity tensors obtained from homogeneous boundary conditions. Further details on the formulation, implementation and application of appropriate boundary conditions in the context of the computational homogenization can be found elsewhere [73-79]. 

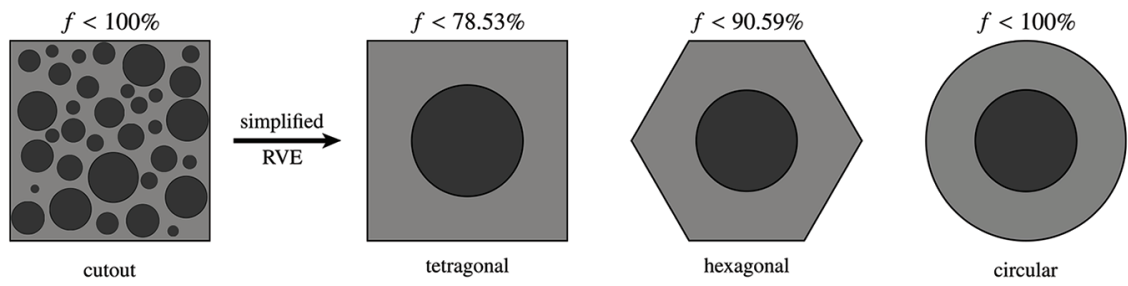

Figure I. Complex representative volume element (RVE) and its simplified counterparts. The inclusion volume fraction at the cutout could reach 100\%. The cutout (left) shall be understood as the RVE. Three simplified RVEs are suggested as sufficient to replace the cutout, namely tetragonal RVE, hexagonal RVE, and circular RVE. For a tetragonal RVE, the maximum reachable volume

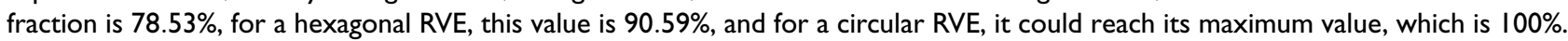
The cutout of the real microstructure can eventually result in isotropic effective behavior. Both tetragonal and hexagonal packings are space-filling, hence intuitively are suitable candidates for a simplified RVE. However, only the circular RVE can furnish isotropic effective behavior resembling the real microstructure. Furthermore, the circular RVE is the only simplified RVE that could reach the maximum volume fraction of a real cutout of the material.

To establish a computational homogenization framework, it is essential to identify a representative volume element (RVE). A proper RVE must be selected such that it contains enough details to sufficiently represent the microstructure of the material and it has to be small enough to be considered as a microstructure surrounded by copies similar to itself, so as to fulfill the assumption of scale separation. In most cases, a proper RVE for a random microstructure is regarded as the smallest possible structure that could sufficiently capture the macroscopic response of the macrostructure. For more details on the definition of the RVE, see Gitman et al. [80], Khisaeva and Ostoja-Starzewski [81], Temizer and colleagues [82, 83], Thomas et al. [84], and Dirrenberger et al. [85], among others. Although small, the RVE could still be too complex for computational homogenization. Thus, instead of studying a complex RVE, one could construct a simpler but statistically similar RVE [86, 87]. Here, the term RVE indicates a simplified microstructure in the sense of a simpler but statistically similar RVE [86, 87] or statistical RVE [88]. In this contribution, we present a systematic study of computational and analytical homogenization by considering three types of RVE; tetragonal, hexagonal, and circular, as shown in Figure 1. Note that the cutout of a real microstructure with a random distribution of particles can eventually result in isotropic effective behavior. While tetragonal and hexagonal packings are space-filling, only the circular RVE can furnish isotropic effective behavior suitable for comparison with analytical solutions here and it is the only RVE that could reach the maximum volume fraction of a real cutout of a material.

The appendix provides a list of definitions for frequently used abbreviations in this manuscript, as well as symbols and notation used to describe the mathematical aspects of the problem. The rest of the manuscript is organized as follows. The theory including the governing equations at both scales, bridging between the scales and satisfying the Hill-Mandel condition via appropriate boundary conditions is presented in Section 2. Material modeling and analytical approaches are detailed in Section 3, followed by a thorough comparison of the numerical and analytical methods to predict the effective response of heterogeneous materials. Section 4 extends the computational homogenization framework to finite deformations; the effects of RVE type and boundary condition are examined. Section 5 summarizes this work and provides further outlook.

\section{Computational homogenization}

This section elaborates on the theoretical aspects of computational homogenization and bridging the scales. The content of this section is fairly standard; however, to set the stage, the important aspects of computational homogenization are briefly reviewed. A detailed exposition of the formulation of computational homogenization and the associated numerical implementation can be found elsewhere $[9,56$, 89-91]. Central to computational homogenization is the separation of the length scales between the micro- and the macroscale. The key idea of computational homogenization is to identify the effective properties at the macroscale through averaging of the microproblem [92-98]. At the microscale, it is 
assumed that the constitutive response of each phase is known. Solving the associated boundary value problem at the microscale and proper averaging over the RVE renders the overall response at the macroscale. The microproblem could be either strain-driven or stress-driven. In strain-driven homogenization, the macroscopic deformation gradient is prescribed and the macroscopic stress is calculated. By contrast, the macroscopic stress is prescribed in stress-driven homogenization for an unknown macroscopic deformation gradient. This contribution is based on first-order strain-driven computational homogenization based on the deformation gradient and the Piola stress. In this section, the macroproblem is formulated first. This is then followed by the microproblem definition. Finally, the micro-to-macro transition and the Hill-Mandel condition are addressed.

\section{I. Macroproblem definition}

Let a macroscopic continuum body take the material configuration ${ }^{\mathrm{M}_{\mathcal{B}}}$ at time $t=0$ and the spatial configuration ${ }^{\mathrm{M}_{t}}$ at time $t>0$, as shown in Figure 2. The boundaries of the body in the material and the spatial configuration are denoted $\partial^{\mathrm{M}} \mathcal{B}_{0}$ and $\partial^{\mathrm{M}} \mathcal{B}_{t}$, respectively. Moreover, ${ }^{\mathrm{M}} \boldsymbol{N}$ and ${ }^{\mathrm{M}} \boldsymbol{n}$ define the material and spatial outward unit normal vectors to the boundaries. The material point ${ }^{\mathrm{M}} \boldsymbol{X}$ is mapped to its spatial counterpart ${ }^{\mathrm{M}} \boldsymbol{x}$ via the nonlinear deformation map ${ }^{\mathrm{M}} \boldsymbol{\varphi}$ as ${ }^{\mathrm{M}} \boldsymbol{x}={ }^{\mathrm{M}} \boldsymbol{\varphi}\left({ }^{\mathrm{M}} \boldsymbol{X}\right)$. The infinitesimal line element $\mathrm{d}^{\mathrm{M}} \boldsymbol{X}$ from the material configuration is mapped to $\mathrm{d}^{\mathrm{M}} \boldsymbol{x}$ in the spatial configuration via the linear map ${ }^{\mathrm{M}} \boldsymbol{F}$ as d ${ }^{\mathrm{M}} \boldsymbol{x}={ }^{\mathrm{M}} \boldsymbol{F} \cdot \mathrm{d}^{\mathrm{M}} \boldsymbol{X}$, where ${ }^{\mathrm{M}} \boldsymbol{F}={ }^{\mathrm{M}} \mathrm{Grad}^{\mathrm{M}} \boldsymbol{\varphi}$ is the macroscopic deformation gradient. In addition, the Jacobian determinant ${ }^{\mathrm{M}} J=\operatorname{det}^{\mathrm{M}} \boldsymbol{F}$ maps the infinitesimal material volume element $\mathrm{d}^{\mathrm{M}} V$ to its spatial counterpart $\mathrm{d}^{\mathrm{M}} v$ via $\mathrm{d}^{\mathrm{M}} v={ }^{\mathrm{M}} J \mathrm{~d}^{\mathrm{M}} V$. Finally, the normal map ${ }^{\mathrm{M}} J^{\mathrm{M}} \boldsymbol{F}^{-\mathrm{t}}$ transforms the directional surface element from the material configuration $\mathrm{d}^{\mathrm{M}} \boldsymbol{S}=\mathrm{d}^{\mathrm{M}} \boldsymbol{S}^{\mathrm{M}} \boldsymbol{N}$ to the directional surface element in the spatial

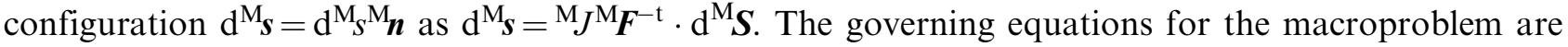
the balances of linear and angular momentum. For a quasistatic case, the balance of linear momentum reads

${ }^{\mathrm{M}} \operatorname{Div}^{\mathrm{M}} \boldsymbol{P}+{ }^{\mathrm{M}} \boldsymbol{b}_{0}^{\mathrm{p}}=0$ in ${ }^{\mathrm{M}} \mathcal{B}_{0},{ }^{\mathrm{M}} \boldsymbol{P} \cdot{ }^{\mathrm{M}} \boldsymbol{N}={ }^{\mathrm{M}} \boldsymbol{t}_{0}$ on $\partial^{\mathrm{M}} \mathcal{B}_{0}$, with ${ }^{\mathrm{M}} \boldsymbol{t}_{0}={ }^{\mathrm{M}} \boldsymbol{t}_{0}^{\mathrm{p}}$ on $\partial^{\mathrm{M}} \mathcal{B}_{0, \mathrm{~N}},{ }^{\mathrm{M}} \boldsymbol{\varphi}_{0}={ }^{\mathrm{M}} \boldsymbol{\varphi}_{0}^{\mathrm{p}}$ on $\partial^{\mathrm{M}} \mathcal{B}_{0, \mathrm{D}}$

where ${ }^{\mathrm{M}} \boldsymbol{b}_{0}^{\mathrm{p}}$ represents the body force density in the material configuration and ${ }^{\mathrm{M}} \boldsymbol{P}$ defines the macroscopic Piola stress. The traction ${ }^{\mathrm{M}_{0}}$ acts on the boundary $\partial^{\mathrm{M}} \mathcal{B}_{0}$. The prescribed traction that acts on the Neumann portion of the boundary $\partial^{\mathrm{M}} \mathcal{B}_{0, \mathrm{~N}} \subset \partial^{\mathrm{M}} \mathcal{B}_{0}$ is denoted ${ }^{\mathrm{M}} \boldsymbol{t}_{0}^{\mathrm{p}}$. The displacement that is applied to

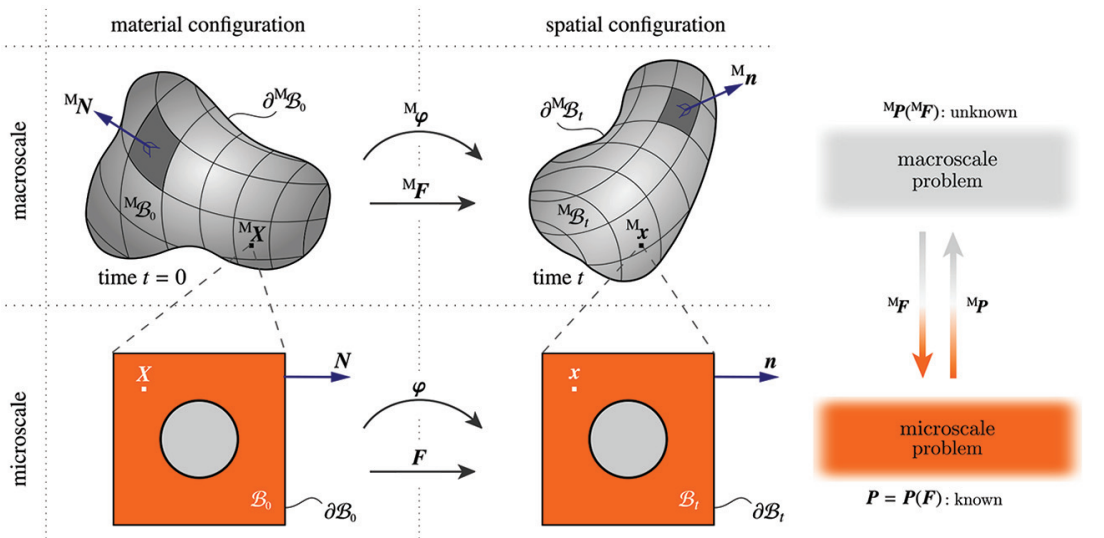

Figure 2. Computational homogenization graphical summary. The material configuration at the macroscale ${ }^{M} \mathcal{B}_{0}$ with the boundary of $\partial^{\mathrm{M}} \mathcal{B}_{0}$ is mapped to its spatial counterpart ${ }^{\mathrm{M}} \mathcal{B}_{t}$ with the boundary of $\partial^{\mathrm{M}} \mathcal{B}_{t}$. The nonlinear map ${ }^{\mathrm{M}} \boldsymbol{\varphi}$ maps points ${ }^{\mathrm{M}} \boldsymbol{X}$ from the material configuration to $M_{\mathbf{X}}$ in the spatial configuration. Line elements are mapped from the material configuration to the spatial configuration via ${ }^{M} \mathbf{F}$. The domain $\mathcal{B}_{0}$ at the microscale corresponds to the representative volume element. In a strain-driven homogenization framework, the macroscopic deformation gradient is the input of the microproblem and the macroscopic Piola stress is obtained by solving the boundary value problem at the microscale. 
the boundary $\partial^{\mathrm{M}} \mathcal{B}_{0}$ is ${ }^{\mathrm{M}} \boldsymbol{\varphi}_{0}$. The prescribed displacement ${ }^{\mathrm{M}} \varphi_{0}^{\mathrm{p}}$ acts on the Dirichlet part of the boundary $\partial^{\mathrm{M}} \mathcal{B}_{0, \mathrm{D}} \subset \partial^{\mathrm{M}} \mathcal{B}_{0}$. The balance of the angular momentum at the macroscale reads

$$
{ }^{\mathrm{M}} \boldsymbol{P} \cdot{ }^{\mathrm{M}} \boldsymbol{F}^{\mathrm{t}}={ }^{\mathrm{M}} \boldsymbol{F} \cdot{ }^{\mathrm{M}} \boldsymbol{P}^{\mathrm{t}},
$$

which is equivalent to the symmetry of the Cauchy stress.

\subsection{Microproblem definition}

As illustrated in Figure 2, the notation for the microproblem mimics that of the macroproblem without the left superscript " $\mathrm{M}$ ". The kinematics of the microproblem, such as points, line elements, surface elements, and volume elements from the material to spatial configuration, read

$$
\boldsymbol{x}=\boldsymbol{\varphi}(\boldsymbol{X}), \quad \mathrm{d} \boldsymbol{x}=\boldsymbol{F} \cdot \mathrm{d} \boldsymbol{X}, \quad \mathrm{d} \boldsymbol{s}=J \boldsymbol{F}^{-\mathrm{t}} \cdot \mathrm{d} \boldsymbol{S}, \quad \mathrm{d} v=J \mathrm{~d} V .
$$

Owing to the scale separation assumption, the body forces vanish at the microscale. The balance of linear momentum for the microproblem holds as

$$
\operatorname{Div} \boldsymbol{P}=0 \text { in } \mathcal{B}_{0}, \boldsymbol{P} \cdot \boldsymbol{N}=\boldsymbol{t}_{0} \text { on } \partial \mathcal{B}_{0}, \quad \text { with } \quad \boldsymbol{t}_{0}=\boldsymbol{t}_{0}^{\mathrm{p}} \text { on } \partial \mathcal{B}_{0, \mathrm{~N}}, \quad \boldsymbol{\varphi}_{0}=\boldsymbol{\varphi}_{0}^{\mathrm{p}} \text { on } \partial \mathcal{B}_{0, \mathrm{D}} .
$$

Finally, the balance of angular momentum reads

$$
\boldsymbol{P} \cdot \boldsymbol{F}^{\mathrm{t}}=\boldsymbol{F} \cdot \boldsymbol{P}^{\mathrm{t}}
$$

\subsection{Micro-to-macro transition}

The micro-to-macro transition is essentially a proper averaging of the quantities at the microscale to link them to their counterparts at the macroscale. The advantage of the micro-to-macro transition is that no assumptions are required regarding the constitutive behavior of the material at the macroscale. This method is capable of incorporating geometrical and physical nonlinearities without additional effort. As depicted in Figure 2, the macroscopic deformation gradient is applied to the microstructure and the microproblem is solved as a classical boundary value problem, using the finite-element method. The macroscopic Piola stress is then computed via proper averaging of the microscopic Piola stress.

The macroscopic deformation gradient and Piola stress are related to their microscopic counterparts via averages over the volume or the boundary of the RVE, as

$$
{ }^{\mathrm{M}} \boldsymbol{F}=\langle\boldsymbol{F}\rangle=\frac{1}{\mathscr{V}_{0}} \int_{\mathcal{B}_{0}} \boldsymbol{F} \mathrm{d} V=\frac{1}{\mathscr{V}_{0}} \int_{\partial \mathcal{B}_{0}} \boldsymbol{\varphi} \otimes \boldsymbol{N} \mathrm{d} A \quad \text { and } \quad{ }^{\mathrm{M}} \boldsymbol{P}=\langle\boldsymbol{P}\rangle=\frac{1}{\mathscr{V}_{0}} \int_{\mathcal{B}_{0}} \boldsymbol{P} \mathrm{d} V=\frac{1}{\mathscr{V}_{0}} \int_{\partial \mathcal{B}_{0}} t_{0} \otimes \boldsymbol{X} \mathrm{d} A .
$$

Moreover, the Hill-Mandel condition necessitates the incremental energy equivalence between the two scales, which reads

$$
{ }^{\mathrm{M}} \boldsymbol{P}: \delta^{\mathrm{M}} \boldsymbol{F}=! \frac{1}{\mathscr{V}_{0}} \int_{\mathcal{B}_{0}} \boldsymbol{P}: \delta \boldsymbol{F} \mathrm{d} V
$$

as the volume average over the RVE. Hill's lemma transforms equation (7) into a surface integral according to

$$
\frac{1}{\mathscr{V}_{0}} \int_{\mathcal{B}_{0}} \boldsymbol{P}: \delta \boldsymbol{F} \mathrm{d} V-{ }^{\mathrm{M}} \boldsymbol{P}: \delta^{\mathrm{M}} \boldsymbol{F}=\int_{\partial \mathcal{B}_{0}}\left[\delta \boldsymbol{\varphi}-\delta^{\mathrm{M}} \boldsymbol{F} \cdot \boldsymbol{X}\right] \cdot\left[\boldsymbol{t}_{0}-{ }^{\mathrm{M}} \boldsymbol{P} \cdot \boldsymbol{N}\right] \mathrm{d} A
$$

Inserting Hill's lemma (equation (8)) into the Hill-Mandel condition (equation (7)) yields

$$
\int_{\partial \mathcal{B}_{0}}\left[\delta \boldsymbol{\varphi}-\delta^{\mathrm{M}} \boldsymbol{F} \cdot \boldsymbol{X}\right] \cdot\left[\boldsymbol{t}_{0}-{ }^{\mathrm{M}} \boldsymbol{P} \cdot \boldsymbol{N}\right] \mathrm{d} A \stackrel{!}{=} 0,
$$


which shall be understood as the Hill-Mandel condition in terms of a surface integral over the boundary of the RVE. Various boundary conditions can fulfill the Hill-Mandel condition a priori [99, 100]. Among all the boundary conditions that satisfy the Hill-Mandel condition, of practical significance are the canonical boundary conditions, that is, the (i) linear DBC, (ii) uniform traction boundary condition (TBC) and (iii) periodic displacement and anti-periodic traction (PBC), imposing

$$
\mathrm{DBC}: \boldsymbol{\varphi}={ }^{\mathrm{M}} \boldsymbol{F} \cdot \boldsymbol{X}, \quad \mathrm{TBC}: \boldsymbol{t}_{0}={ }^{\mathrm{M}} \boldsymbol{P} \cdot \boldsymbol{N}, \quad \text { and } \mathrm{PBC}:\left[\boldsymbol{\varphi}-{ }^{\mathrm{M}} \boldsymbol{F} \cdot \boldsymbol{X}\right] \text { and }\left[\boldsymbol{t}_{0}-{ }^{\mathrm{M}} \boldsymbol{P} \cdot \boldsymbol{N}\right],
$$

on the boundary of the RVE. For strain-driven computational homogenization, here the condition ${ }^{\mathrm{M}} \boldsymbol{F}=\langle\boldsymbol{F}\rangle$ is a priori satisfied for both the DBC and the PBC. However, this is not the case for the TBC and the condition ${ }^{\mathrm{M}} \boldsymbol{F}=\langle\boldsymbol{F}\rangle$ shall be regarded as a constraint. It is noteworthy that all these boundary conditions satisfy the balance of angular momentum at the macroscale. It is commonly accepted that the $\mathrm{DBC}$ and the $\mathrm{TBC}$ overestimate and underestimate the $\mathrm{PBC}$, respectively.

\section{Numerical versus analytical homogenization}

In this section, first the material modeling of the problem is presented. This is then followed by a brief review of the analytical methods developed to determine the overall response of composites for smallstrain linear elasticity. Finally, a thorough comparison of numerical and analytical results for various RVE types is provided.

\section{I. Material modeling}

The constituents of heterogeneous materials at the microscale are assumed to be hyperelastic with known behavior. The bonding between the matrix and the inclusion is considered perfect. Both matrix and inclusion behave according to the free energy density

$$
\psi(\boldsymbol{F})=\frac{1}{2} \mu[\boldsymbol{F}: \boldsymbol{F}-2-2 \log J]+\frac{1}{2} \lambda \log ^{2} J
$$

associated with a neo-Hookean response with Lamé parameters $\lambda$ and $\mu$, whereby the bulk modulus associated with the plane-strain condition of interest here relates to the Lamé parameters as $\kappa=\mu+\lambda$. Using the Coleman-Noll procedure, the Piola stress is derived as

$$
\boldsymbol{P}=\frac{\partial \psi}{\partial \boldsymbol{F}}=\mu\left[\boldsymbol{F}-\boldsymbol{F}^{-\mathrm{t}}\right]+\lambda \log J \boldsymbol{F}^{-\mathrm{t}} .
$$

The corresponding fourth-order Piola tangent tensor reads

$$
\mathbb{A}=\frac{\partial \boldsymbol{P}}{\partial \boldsymbol{F}}=\mu\left[\boldsymbol{I} \otimes \boldsymbol{I}+\boldsymbol{F}^{-\mathrm{t}} \underline{\otimes \boldsymbol{F}^{-1}}\right]+\lambda\left[\boldsymbol{F}^{-\mathrm{t}} \otimes \boldsymbol{F}^{-\mathrm{t}}-\log J \boldsymbol{F}^{-\mathrm{t}} \underline{\otimes \boldsymbol{F}^{-1}}\right] .
$$

The nonstandard tensor products $\bar{\otimes}$ and $\underline{\otimes}$ of two second-order tensors $\boldsymbol{A}$ and $\boldsymbol{B}$ are the forth-order tensors $\mathbb{D}=\boldsymbol{A} \bar{\otimes} \boldsymbol{B}$ and $\mathbb{C}=\boldsymbol{A} \otimes \boldsymbol{B}$, respectively, with the components $D_{i j k l}=A_{i k} B_{j l}$ and $C_{i j k l}=A_{i l} B_{j k}$.

Small-strain linear elasticity relates the stress $\sigma$ to the strain $\varepsilon$ according to the linear relation $\sigma=\mathbb{C}: \varepsilon$ in which the strain $\varepsilon$ and the fourth-order constitutive tensor $\mathbb{C}$ can be related to their counterparts at finite deformations via

$$
\varepsilon=\frac{1}{2}\left[\boldsymbol{F}^{\mathrm{t}}+\boldsymbol{F}\right]-\boldsymbol{I} \quad \text { and } \quad \mathbb{C}=\left.\mathbb{A}\right|_{\boldsymbol{F}=\boldsymbol{I}}=\mu[\overline{\boldsymbol{I} \otimes} \boldsymbol{I}+\boldsymbol{I} \otimes \underline{I}]+\lambda[\boldsymbol{I} \otimes \boldsymbol{I}],
$$

and the stress $\boldsymbol{\sigma}$ reads

$$
\boldsymbol{\sigma}=\operatorname{Lin} \boldsymbol{P}=\left.\boldsymbol{P}\right|_{\boldsymbol{F}=\boldsymbol{I}}+\left.\mathbb{A}\right|_{\boldsymbol{F}=\boldsymbol{I}}:[\boldsymbol{F}-\boldsymbol{I}]=2 \mu \varepsilon+\lambda[\varepsilon: \boldsymbol{I}] \boldsymbol{I}
$$




\subsection{Analytical estimates at small strains}

Analytical methods in homogenization have been established to derive a relation for the overall response of heterogeneous media. Analytical methods are based on certain simplifying assumptions so as to achieve an explicit analytical solution. In this section, the most significant and extensively used analytical estimates are briefly listed. Throughout this manuscript, the matrix and inclusion properties are identified by the subscripts 1 and 2, respectively.

Voigt bounds correspond to a uniform strain field within the RVE resulting in the upper limit for the effective overall response of the material as

$$
\kappa=[1-f] \kappa_{1}+f \kappa_{2} \text { and } \mu=[1-f] \mu_{1}+f \mu_{2},
$$

where $f$ is the inclusion volume fraction. Note that the uniform-strain assumption violates the balance of linear momentum, in general. Thus, Voigt bounds shall only be understood as an upper unreachable bound. Reuss bounds correspond to a uniform stress field within the RVE, leading to the lower limit for the effective response of the material as

$$
\kappa=\frac{\kappa_{1} \kappa_{2}}{[1-f] \kappa_{2}+f \kappa_{1}} \quad \text { and } \quad \mu=\frac{\mu_{1} \mu_{2}}{[1-f] \mu_{2}+f \mu_{1}} .
$$

The uniform-stress assumption violates the compatibility of the strain field; thus, Reuss bounds shall only be understood as a lower unreachable bound.

Hashin and Rosen [24] proposed a predictive model based on a composite cylinder assemblage (CCA) to obtain the bulk and shear moduli of transversely isotropic composites having circular inclusions in hexagonal and random arrays. The effective coefficients in this approach are frequently used. In this method, the upper and lower bounds on the bulk modulus coincide and read

$$
\kappa_{\mathrm{U}}=\kappa_{\mathrm{L}}=\frac{\kappa_{1}\left[\mu_{1}+\kappa_{2}\right]+f \mu_{1}\left[\kappa_{2}-\kappa_{1}\right]}{\mu_{1}+\kappa_{2}+f\left[\kappa_{1}-\kappa_{2}\right]},
$$

where the subscripts "U" and "L" denote the upper and the lower bounds, respectively. While the mathematical procedure for the determination of bounds for the shear modulus has been addressed precisely [24], closed-form expressions for the bounds for the shear modulus, for the first time, to our knowledge, are given here as

$$
\left.\begin{array}{rl}
\left.\left.\mu_{\mathrm{U}}=\mu_{1}\left[\frac{2 f\left[\kappa_{1}+\mu_{1}\right]\left[\frac{\mu_{2}-\mu_{1}}{\mu_{2} \kappa_{1}+\mu_{1} \kappa_{1}+2 \mu_{1} \mu_{2}}\right]}{3[1-f]^{2}\left[\frac{\kappa_{1}}{\kappa_{1}+2 \mu_{1}}\right]^{2}}\right]-1\right]+1\right] & \frac{f\left[\mu_{2}-\mu_{1}\right]\left[\kappa_{1}+2 \mu_{1}\right]}{\mu_{2} \kappa_{1}+\mu_{1} \kappa_{1}+2 \mu_{1} \mu_{2}}\left[\frac{f^{3}\left[\frac{\left.\mu_{2} \kappa_{2}\left[\kappa_{1}+2 \mu_{1}\right]-\mu_{1} \kappa_{1}\left[\kappa_{2}+2 \mu_{2}\right]\right]}{\left[\kappa_{1}+2 \mu_{1}\right]\left[\kappa_{2} \mu_{2}+\mu_{1}\left[\kappa_{2}+2 \mu_{2}\right]\right]}\right]-1}{\mu_{\mathrm{L}}}\right]
\end{array}\right],
$$


Shortly afterwards, Hashin [101] used the variational approach of Hashin and Shtrikman [23] to derive bounds on the overall response of fiber composites with transverse isotropy. The aim of this approach was to tighten the bounds proposed by Reuss and Voigt. The Hashin-Shtrikman bounds for the overall bulk and shear moduli read

$$
\begin{aligned}
\kappa_{\mathrm{U}}=\kappa_{1}+\frac{f}{\frac{1}{\kappa_{2}-\kappa_{1}}+\frac{1-f}{\kappa_{1}+\mu_{1}}}, & \kappa_{\mathrm{L}}=\kappa_{2}+\frac{[1-f]}{\frac{1}{\kappa_{1}-\kappa_{2}}+\frac{f}{\kappa_{2}+\mu_{2}}}, \\
\mu_{\mathrm{U}}=\mu_{1}+\frac{1}{\frac{1}{\mu_{2}-\mu_{1}}+\frac{[1-f]\left[\kappa_{1}+2 \mu_{1}\right]}{2 \mu_{1}\left[\kappa_{1}+\mu_{1}\right]}}, & \mu_{\mathrm{L}}=\mu_{2}+\frac{\frac{1}{\frac{1-f]}{\mu_{1}-\mu_{2}}+\frac{f\left[\kappa_{2}+2 \mu_{2}\right]}{2 \mu_{2}\left[\kappa_{2}+\mu_{2}\right]}},}{},
\end{aligned}
$$

for stiffness ratios less than one. The upper and the lower bound switch for stiffness ratios greater than one.

The Hashin and Shtrikman variational principle $[23,101]$ is well known to provide the best bounds, independent of the phase geometry, and are formulated only in terms of the phase properties and inclusion volume fraction. Hill [25] also derived bounds on five different effective properties of composites with transversely isotropic geometry. Walpole [27, 102] utilized piecewise uniform polarization and rederived these bounds in a more general fashion. Walpole's method also includes anisotropic constituents and disk-shape fiber composites. All aforementioned bounds were independent of phase geometry and they were only applicable on three types of geometries; laminated, isotropic, and transversely isotropic. Later, Willis [29] manipulated the Hashin-Shtrikman bounds by inserting the two-point correlation function to account for more general cases of phase geometry. If the two-point correlation function involves radial, cylindrical, or disk symmetry, these bounds could be readily recovered. See Weng [103] for more details on the derivation of explicit forms for the Willis bounds.

\subsection{Numerical examples}

This section provides a comprehensive comparison between the analytical estimates and computational results through a series of numerical examples for tetragonal, hexagonal, and circular RVEs. Figure 3 shows the packing of these RVEs. The area of each RVE is set to 1 so that the inclusion area corresponds to the volume fraction $f$. Obviously, the volume fraction $f$ could not exceed a certain value for the tetragonal and hexagonal RVEs, see Figure 4. All the examples are solved using our in-house finiteelement code and discretized using biquadratic Lagrange elements.

To provide a thorough and systematic comparison of the numerical and analytical results, the overall bulk modulus $\mathrm{M}_{\kappa}$, shear modulus $\mathrm{M}_{\mu}$, and Poisson ratio $\mathrm{M}_{\nu}$ of transversely isotropic composites are examined. Figure 5 clearly illustrates all the cases investigated in what follows. Five different stiffness ratios of $0.01,0.1,1,10$, and 100 are considered for each $\mathrm{RVE}$. The stiffness ratio represents the ratio of the inclusion to matrix (incl./matr.) Lamé parameters. A stiffness ratio less than one corresponds to a more compliant inclusion within the matrix; in the limit of incl./matr. $\rightarrow 0$, the inclusion represents a void. By contrast, a stiffness ratio greater than one corresponds to a stiffer inclusion compared with the
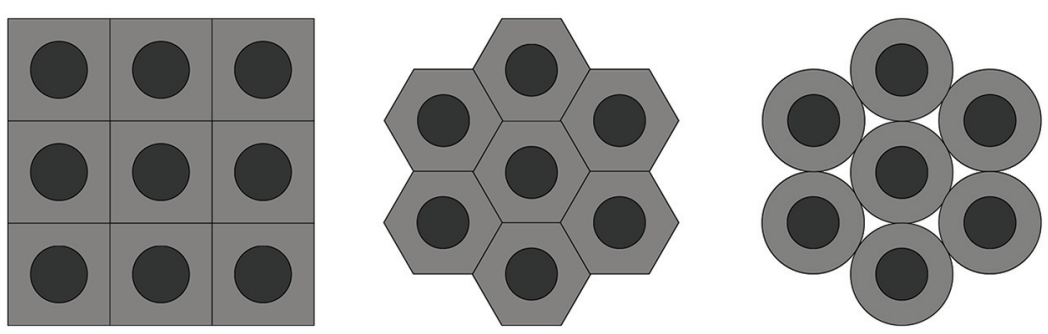

Figure 3. Packing network for each representative volume element (RVE): left, tetragonal RVE packing; center, hexagonal RVE packing; right, circular RVE packing. The boundaries of the tetragonal and hexagonal RVEs completely match. 


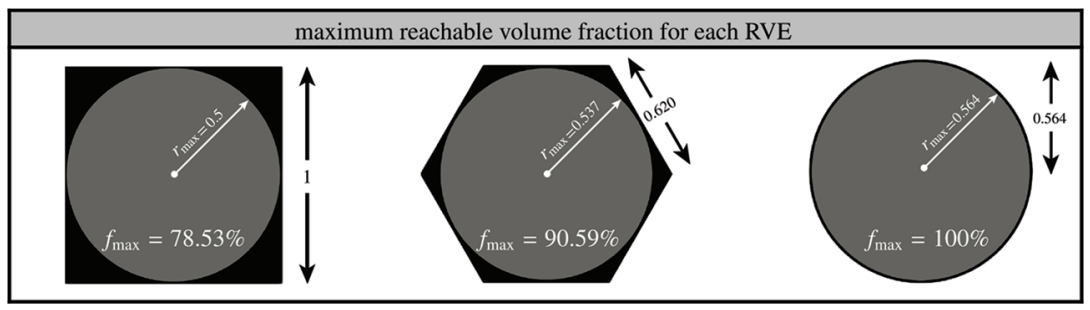

Figure 4. Maximum reachable volume fraction for each representative volume element (RVE). For the circular RVE, the inclusion can embed the whole, owing to its geometry.

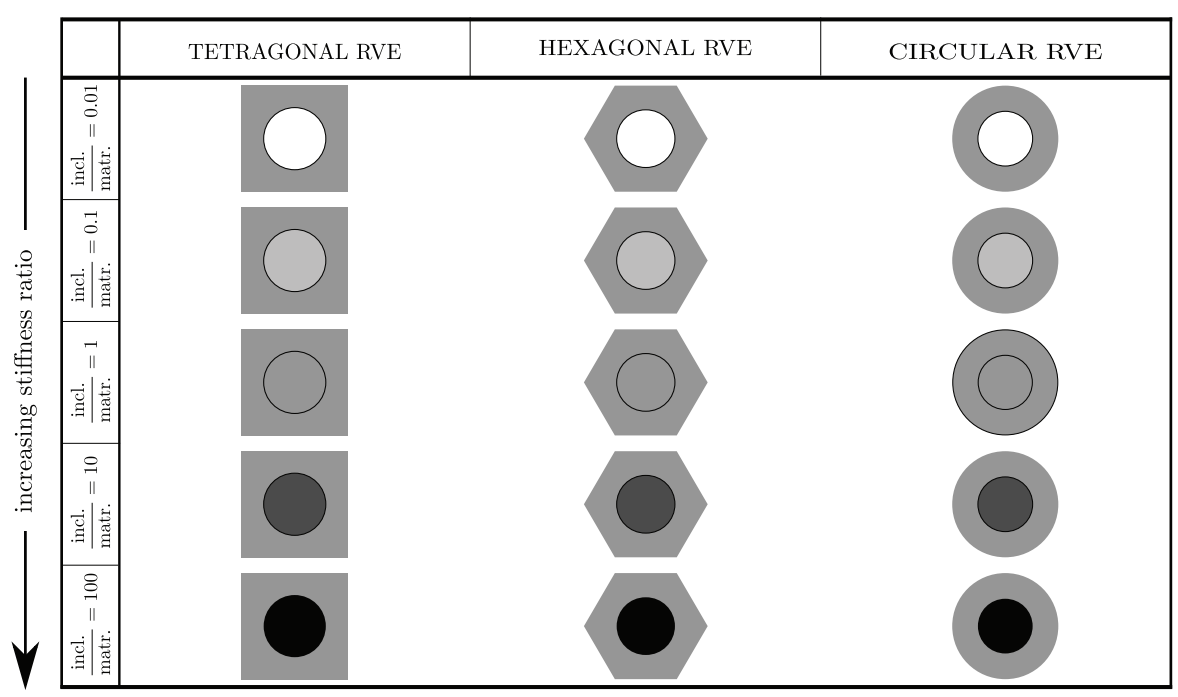

Figure 5. Different stiffness ratios for various representative volume element (RVE) types. Columns correspond to RVE types and rows correspond to stiffness ratios. Matrix parameters remain the same throughout the examples, while inclusion parameters vary in order to produce the required stiffness ratios. The stiffness of the inclusion is identified by a color scheme varying from light to dark.

matrix and in the limit of incl./matr. $\rightarrow \infty$, the inclusion acts as a rigid fiber. Throughout all the examples, the matrix properties are set to $\lambda_{1}=10, \mu_{1}=10$, while the inclusion parameters vary to generate the predefined stiffness ratios.

Figure 6 shows the effective bulk modulus ${ }^{\mathrm{M}_{\kappa}}$ with respect to the volume fraction $f$ for all the cases depicted in Figure 5. For tetragonal and hexagonal RVEs, five individual lines represent the analytical estimates of Voigt and Reuss, together with the numerical results corresponding to the $\mathrm{DBC}, \mathrm{PBC}$, and TBC. The RVEs in tetragonal and hexagonal packings are space-filling but cannot capture the isotropic behavior of the effective material. By contrast, the circular RVE renders isotropic behavior; thus, comparison with the Hashin-Shtrikman and CCA approaches is justifiable. Voigt and Reuss bounds always provide reliable bounds. As expected, the PBC is bounded by the TBC from below and the DBC from above. Moving from the tetragonal RVE toward the circular RVE, we observe that the numerical results tend to converge until they totally coincide at the circular RVE. Therefore, different boundary conditions result in the same responses when the RVE is circular. A smaller difference between the numerical results is observed for small volume fractions. As previously mentioned, for the expansion case, the CCA upper and lower bounds coincide, while the Hashin-Shtrikman bounds (HSBs) do not. For incl./matr. $<1$, a remarkable agreement is observed between the numerical results, CCA, and upper HSB, and for incl./matr. $>1$, the numerical results, CCA, and lower HSB coincide. For incl./matr. = 1, all the results coincide, since the domain is uniform.

Figure 7 shows the effective shear modulus ${ }^{\mathrm{M}} \mu$ versus the volume fraction for different RVEs and stiffness ratios. It is observed that different boundary conditions, in general, provide more 


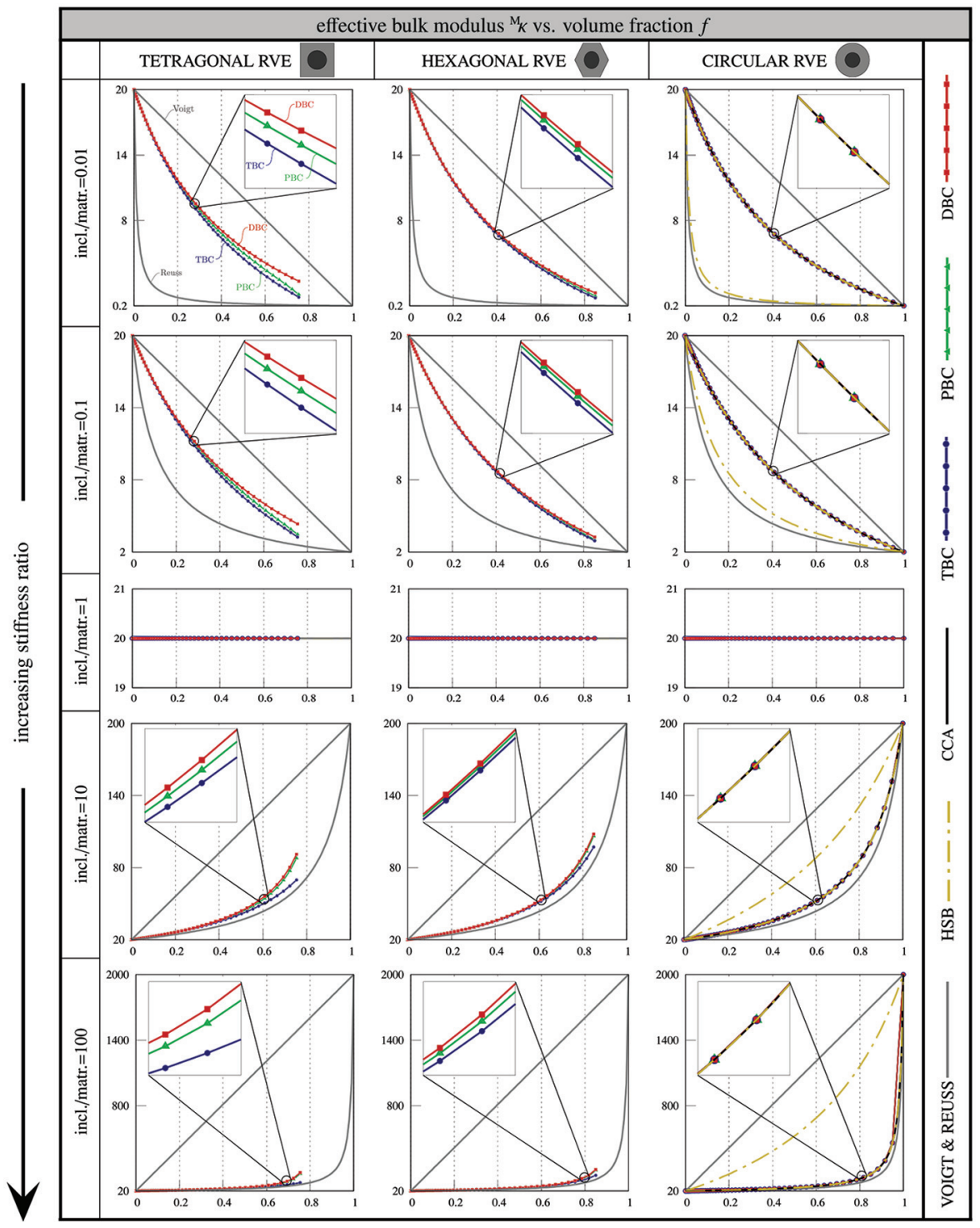

Figure 6. Effective bulk modulus versus volume fraction $f$. The enlargements in the insets provide more clarity when the results are less distinguishable. The numerical results are shown by lines with points on top of them, whereas the analytical results are shown using lines solely.

CCA: composite cylinder assemblage; DBC: displacement boundary condition; HSB: Hashin-Shtrikman bound; PBC: periodic boundary condition; RVE: representative volume element; TBC: traction boundary condition.

distinguishable effective values than the previous case. Nevertheless, as we move from the tetragonal RVE toward the circular RVE, the PBC moves toward the DBC and ultimately coincides with it. Thus, prescribing $\mathrm{PBC}$ and $\mathrm{DBC}$ to the circular RVE yield identical responses. The PBC renders the most sensitive numerical result to the RVE type for shear deformation. In contrast with the previous case, there is no coincidence between the CCA and HSBs. A striking agreement is observed between the CCA lower bound and the TBC and between the CCA upper bound and the $\mathrm{DBC}$ or PBC. A counterintuitive observation is that for incl./matr. $<1$, the DBC and the PBC, together with the CCA upper bound, overestimate the upper HSB, and for incl./matr. $>1$, the TBC, together with the CCA lower bound, underestimate the lower HSB. This observation shall be compared with Hashin's remark [104] that it has never been shown that his bounds on shear modulus are the best possible bounds. Clearly, we observe that his bounds on shear modulus do not serve as "bounds," at least not for CCA in the sense of the Voigt and Reuss bounds. 


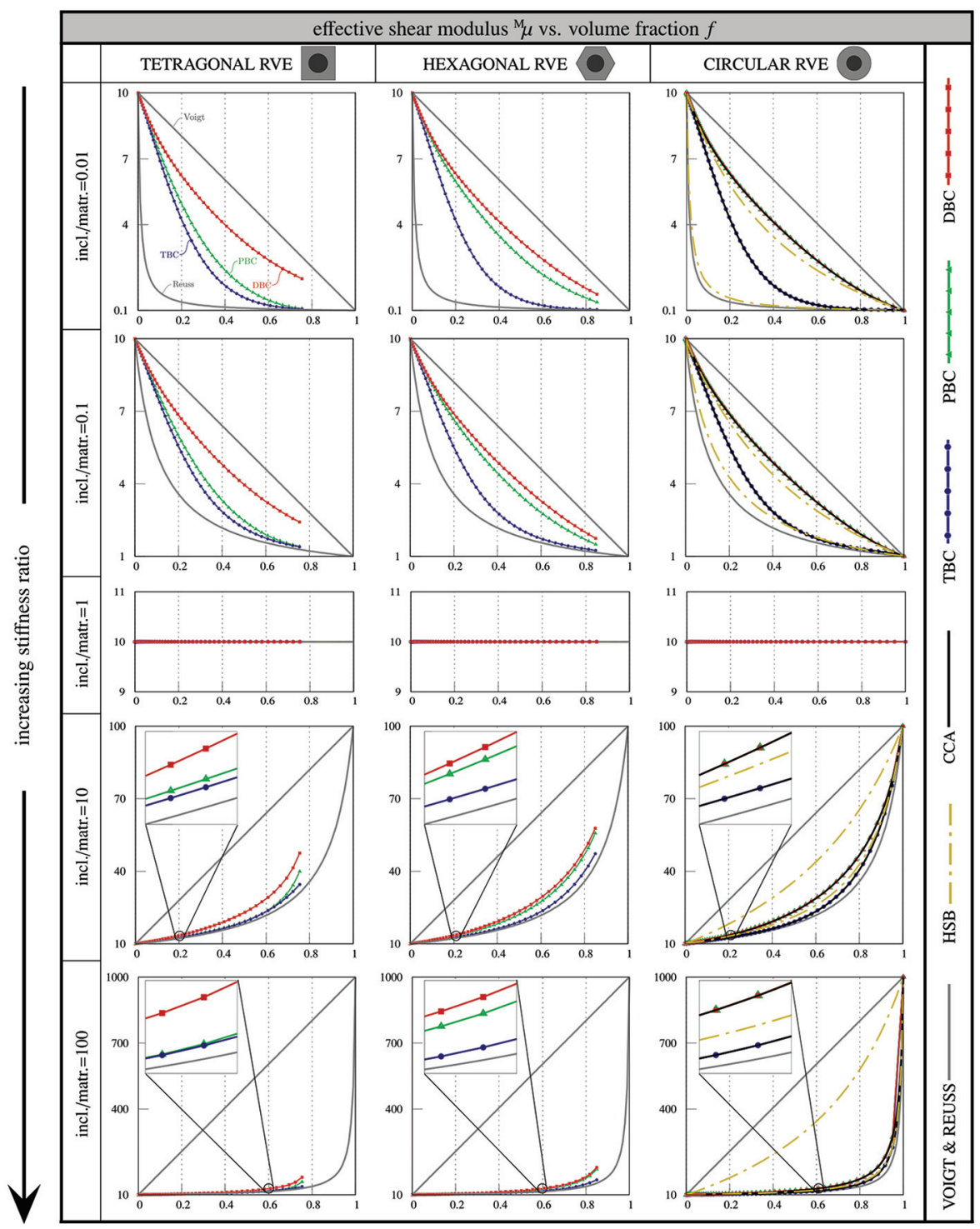

Figure 7. Effective shear modulus versus volume fraction $f$. The enlargements in the insets are included to provide more clarity when the results are less distinguishable. The numerical results are shown by lines with points on top of them, whereas the analytical results are shown using lines solely.

CCA: composite cylinder assemblage; DBC: displacement boundary condition; HSB: Hashin-Shtrikman bound; PBC: periodic boundary condition; RVE: representative volume element; TBC: traction boundary condition.

So far, we have studied the effects of the variation of the volume fraction on the overall macroscopic response. Figure 8 demonstrates the variation of the effective moduli with respect to the stiffness ratio. Two different volume fractions, $f=25 \%$ and $f=75 \%$, are considered, corresponding to the top row and the bottom row, respectively. As expected, all the results coincide at incl./matr. $=1$. The gap between the results widens as the stiffness ratio recedes from one. The difference between the numerical and analytical results is more distinct for the shear modulus $\mathrm{M}_{\mu}$ than for the bulk modulus $\mathrm{M}_{\kappa}$. We observe better agreement between the numerical results for the hexagonal RVE than for the tetragonal RVE. For the bulk modulus, the transition of the numerical results coinciding with the upper and lower HSBs for different stiffness ratios is more obvious in this figure. Another shortcoming of the HSBs is that, like Voigt and Reuss bounds, they cannot distinguish between the matrix and the fiber. Figure 9 sheds light on this issue by providing a comparison of the analytical estimates and the numerical results obtained using the circular RVE. The first column corresponds to certain properties for the matrix and 


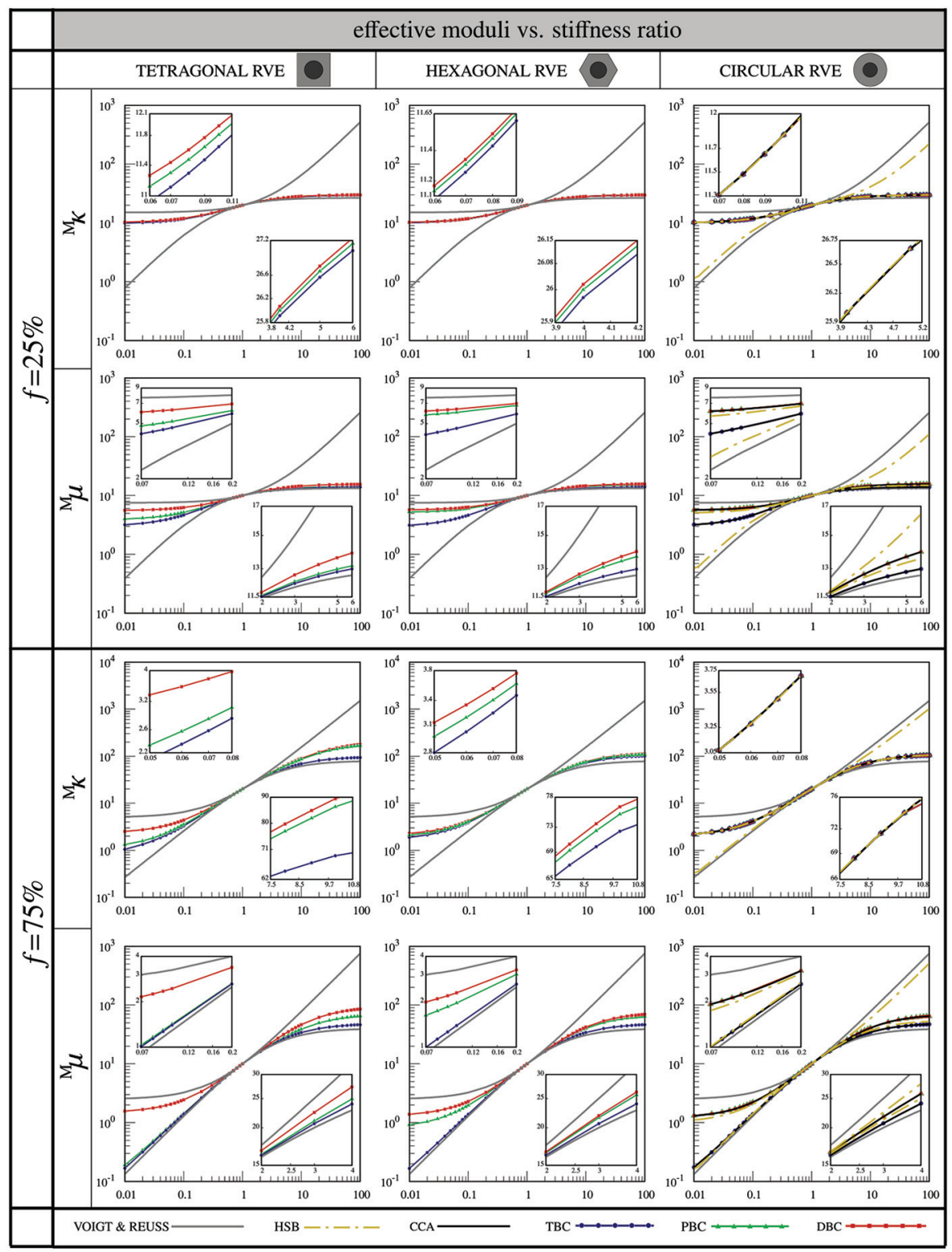

Figure 8. Overall moduli versus stiffness ratio in a logarithmic scale. Two specific volume fractions are considered and are represented in each row. A good agreement of the results is observed for the volume fraction $f=25 \%$. Increasing the volume fraction to $f=75 \%$ leads to more distinct results. More accordance between the results is observed for the bulk modulus ${ }^{M} \kappa$ than for the shear modulus ${ }^{M} \mu$. The enlargements in the insets provide for further clarity.

CCA: composite cylinder assemblage; DBC: displacement boundary condition; HSB: Hashin-Shtrikman bound; PBC: periodic boundary condition; RVE: representative volume element; TBC: traction boundary condition.

fiber. In the second column, the properties are switched and the results are illustrated with respect to matrix volume fraction $(1-f)$. The third column shows the subtraction of the results associated with the first and second columns. We observe that, by contrast with the numerical results and the CCA, the Voigt bounds, Reuss bounds, and HSBs are incapable of distinguishing between the matrix and the fiber; hence, the difference between the responses vanishes in the right column. Figure 10 illustrates the numerical results of various material properties with respect to the volume fraction for the circular RVE. As observed previously, when the RVE is circular, the DBC and PBC always render identical responses. For the bulk modulus, the $\mathrm{DBC}$ coincides with the $\mathrm{TBC}$, while for the shear modulus, the TBC underestimates the DBC, as expected. Somewhat strikingly, for the Poisson ratio, the TBC 


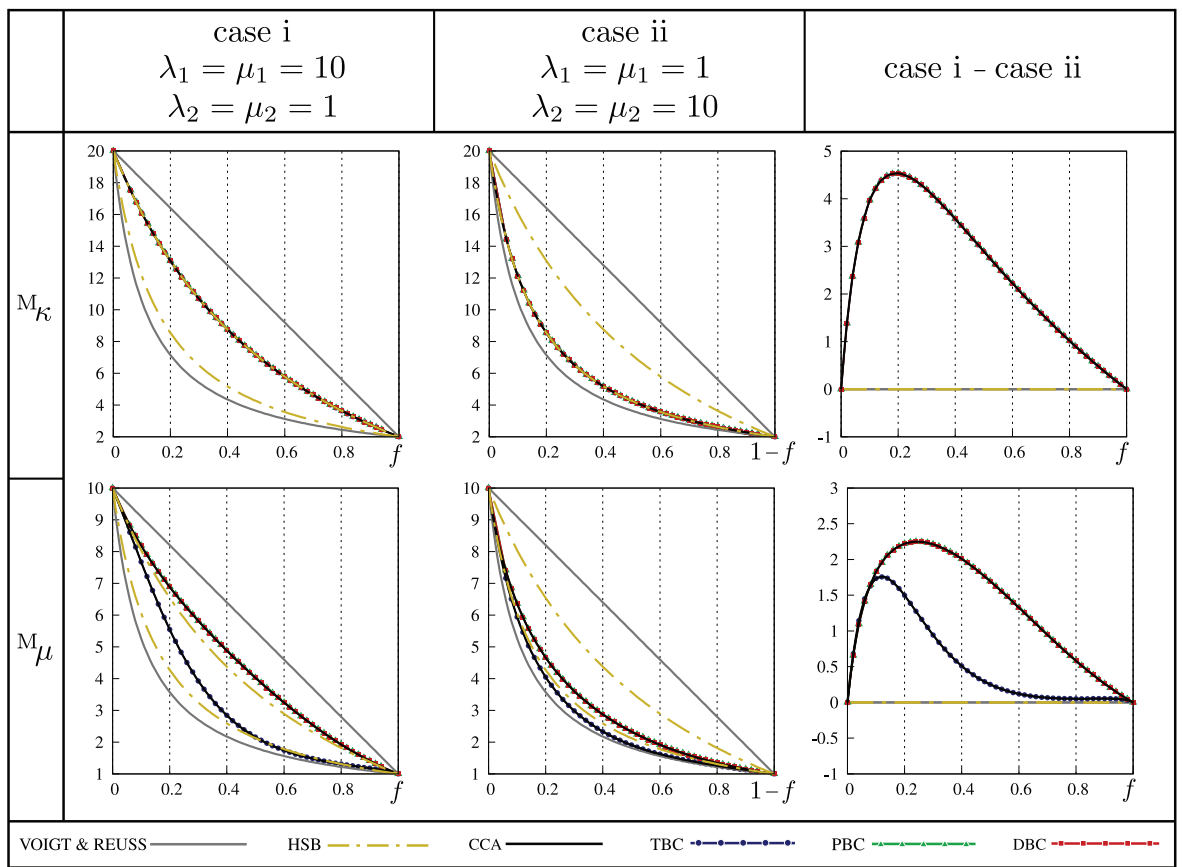

Figure 9. Insensitivity of the Hashin-Shtrikman bounds to the microstructure's constituents. Similarly, Voigt and Reuss bounds do not distinguish between the fiber and the matrix. The numerical results depicted in this figure correspond to the circular representative volume element. The composite cylinder solution totally agrees with the numerical results. The first column shows incl. $/$ matr $=0.1$, where the inclusion and the matrix Lamé parameters are set to I and I0, respectively, and the fiber's volume fraction is $f$. The second column corresponds to incl./matr $=10$, where the inclusion and the matrix Lamé parameters are set to 10 and $I$, respectively, and the $x$-axis shows $(I-f)$ instead of $f$. The third column shows the subtraction of the results in the first and the second column.

CCA: composite cylinder assemblage; DBC: displacement boundary condition; HSB: Hashin-Shtrikman bound; PBC: periodic boundary condition; TBC: traction boundary condition.

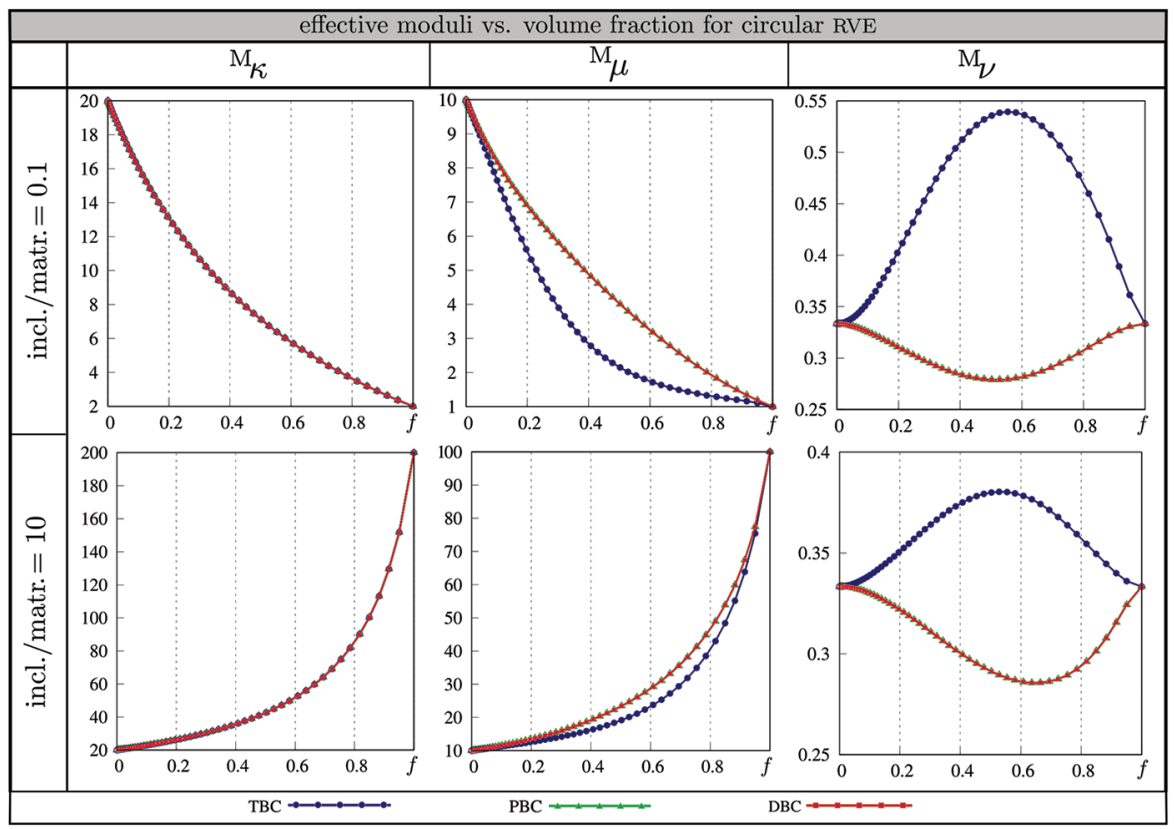

Figure 10. Different moduli versus volume fraction for two different stiffness ratios. The numerical results depicted in this figure correspond to the circular representative volume element. Each column corresponds to a specific material property and each row stands for a specific stiffness ratio. Only numerical results are included for the Poisson ratio, since analytical estimates do not provide physically meaningful results.

DBC: displacement boundary condition; PBC: periodic boundary condition; RVE: representative volume element; TBC: traction boundary condition. 
overestimates the DBC. Another counterintuitive observation is that although the Poisson ratio of the fiber and matrix are identical, the overall Poisson ratio is dependent on the fiber volume fraction and is not constant.

Heterogeneous materials generally possess a periodic or random composition to some extent. Clearly, the distribution pattern of the inclusions influences the overall material response [105, 106]. The next set of numerical studies aims to highlight the effects of different morphologies of the microstructures on the effective properties. To do this, we consider several RVEs with identical volume fractions of $f=15 \%$ and with random and periodic distribution of inclusions, under the three canonical boundary conditions. The periodic microstructure is modeled such that the inclusions of the same size are uniformly distributed throughout the RVE, whereas the random microstructures contain inclusions with different sizes and no specific order. The numerical results corresponding to the circular RVE with the same volume fraction, as well as the CCA and HSBs are also included, for the sake of completeness. This study is performed for two stiffness ratios of 0.1 and 10 and the results are depicted in Figures 11 and 12. The

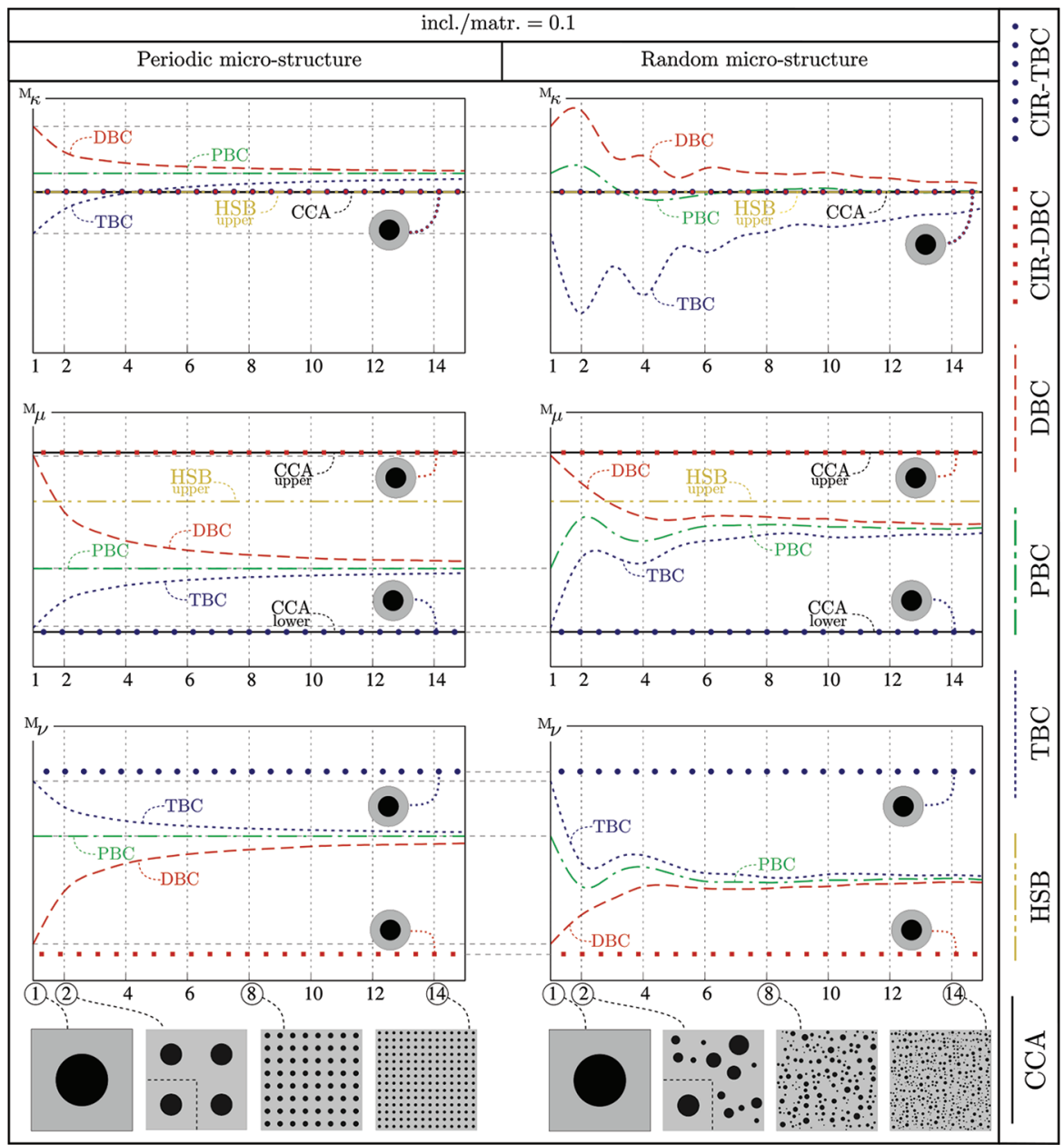

Figures I I. Evolution of the effective properties versus the degree of periodicity and randomness for periodic and random macrostructures. Three different boundary conditions are imposed to two different microstructures having different distributions of inclusions for a stiffness ratio of 0 . I. The volume fraction is set to $15 \%$. The size of the representative volume element remains constant as the level of periodicity or randomness is increased. The horizontal axis shows the degree of periodicity and randomness for the periodic and random microstructure, respectively. The microstructures for some levels are depicted at the bottom of the figure. Analytical bounds, as well as numerical results for circular representative volume elements, are included, to provide further information.

CCA: composite cylinder assemblage; CIR: circular; DBC: displacement boundary condition; HSB: Hashin-Shtrikman bound; PBC: periodic boundary condition; RVE: representative volume element; TBC: traction boundary condition. 


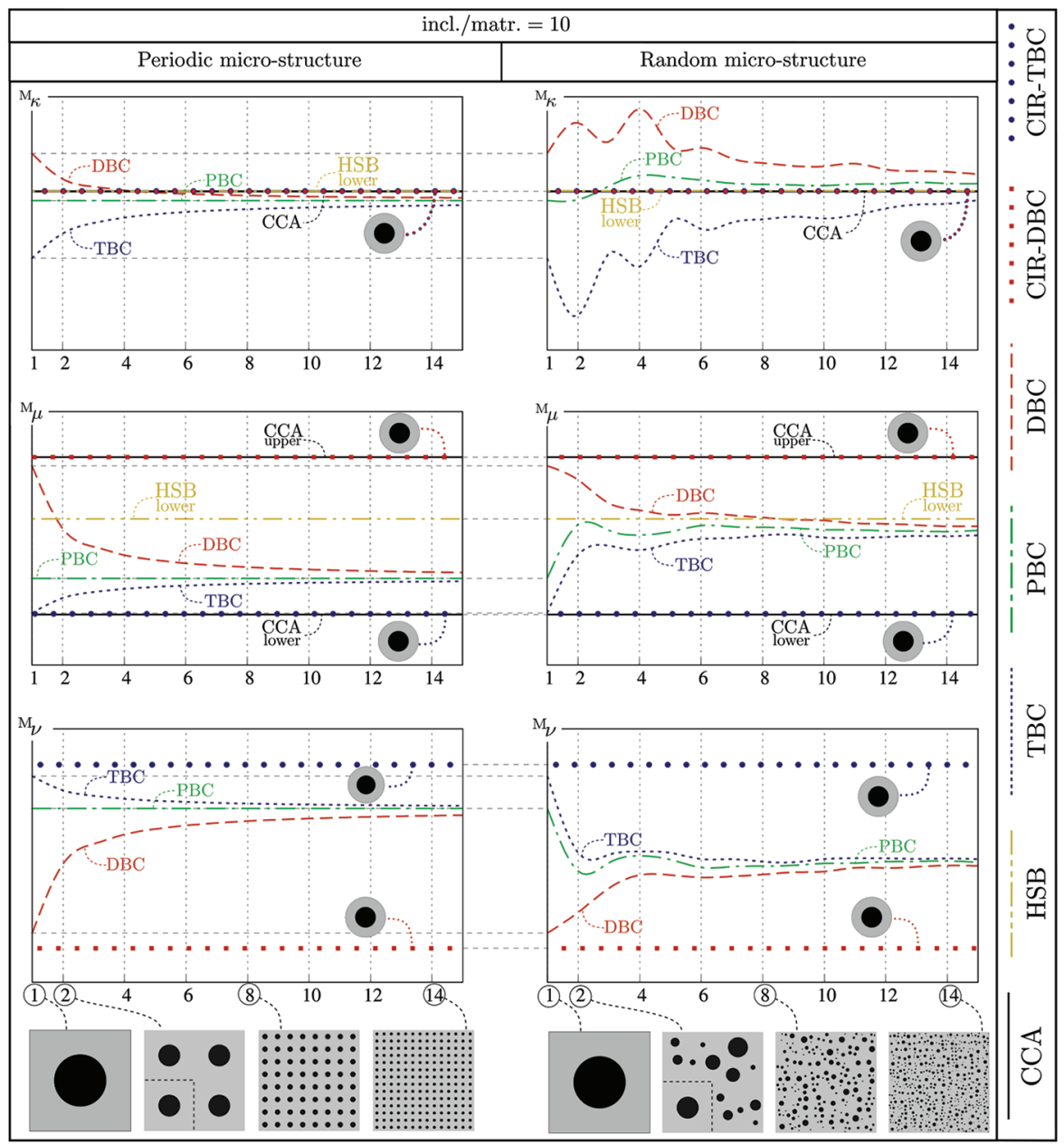

Figures 12. Evolution of the effective properties versus the degree of periodicity and randomness for periodic and random macrostructures. Three different boundary conditions are imposed to two different microstructures having different distribution of inclusions for a stiffness ratio of 10 . The volume fraction is set to $15 \%$. The size of the representative volume element remains constant as the level of periodicity or randomness is increased. The horizontal axis shows the degree of periodicity and randomness for the periodic and random microstructure, respectively. The microstructures for some levels are depicted at the bottom of the figure. Analytical bounds, as well as numerical results for circular representative volume elements, are included, to provide further information.

CCA: composite cylinder assemblage; CIR: circular; DBC: displacement boundary condition; HSB: Hashin-Shtrikman bound; PBC: periodic boundary condition; RVE: representative volume element; TBC: traction boundary condition.

variation of the effective bulk modulus, shear modulus, and Poisson ratio with respect to increasing numbers of inclusions within the microstructure are examined. The lower HSB in Figure 11 and the upper HSB in Figure 12 are eliminated, since they do not fit within the given range. We shall highlight that for each level of the random microstructure, almost 10 samples with different distribution patterns are investigated. That is, the effective responses shown in Figures 11 and 12 do not correspond only to the microstructures depicted at the bottom but reflect the average of the effective responses obtained from 10 samples.

In both types of microstructure and for both stiffness ratios, the results from the $\mathrm{DBC}, \mathrm{PBC}$, and TBC tend to converge to an effective response as the number of inclusions increases sufficiently. This trend is smoother for the periodic microstructure than for the random microstructure, where some fluctuations are present. We observe that, owing to the periodicity of the periodic microstructure, the PBC remains constant, and the $\mathrm{TBC}$ and $\mathrm{DBC}$ tend to approach to it from below and above, respectively. 


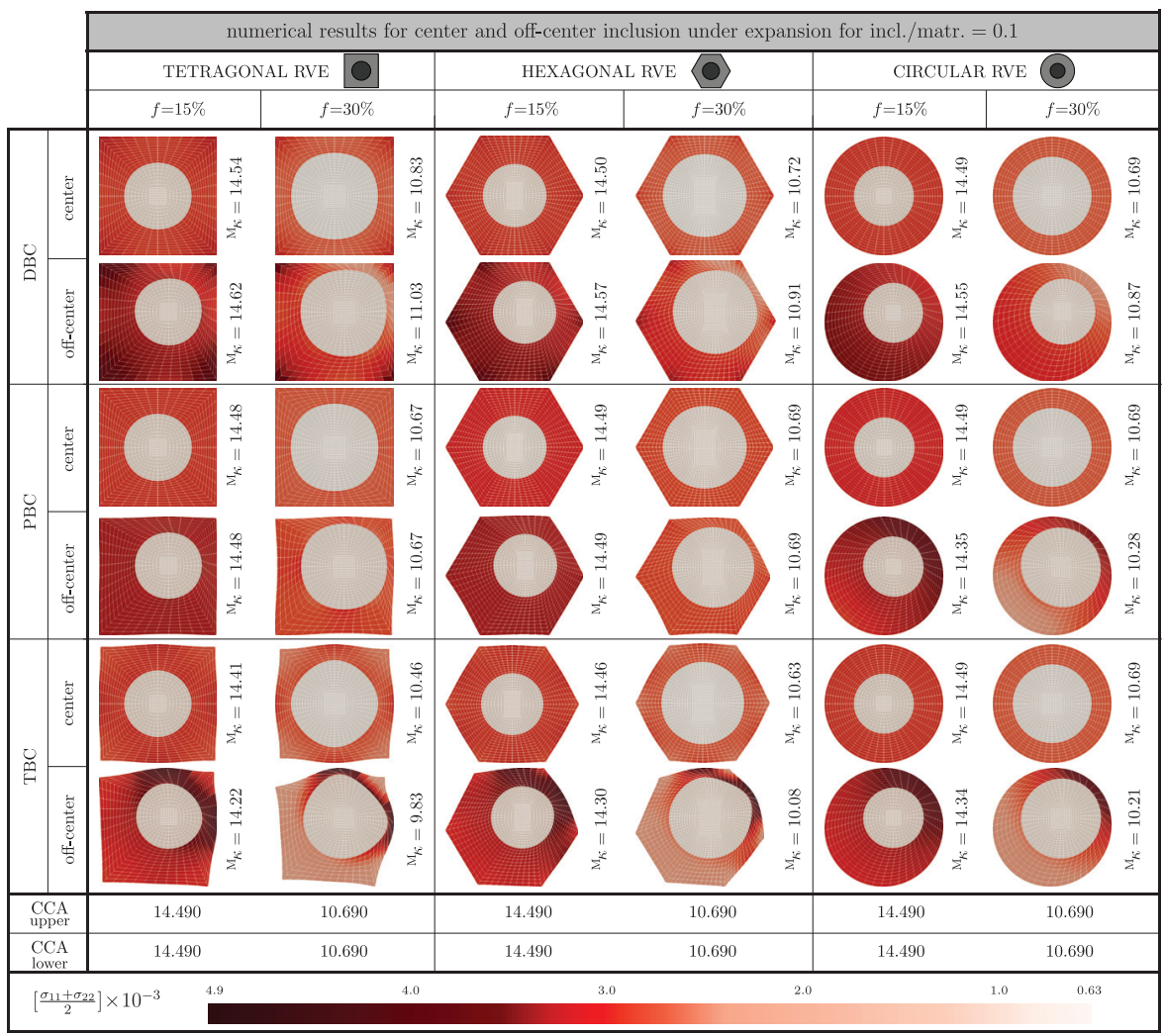

Figure 13. Stress distribution within the RVE under volumetric expansion for both center and off-center inclusions when the inclusion is more compliant than the matrix (incl./matr. $=0.1$ ). The distribution of the stress component $\left[\sigma_{11}+\sigma_{22}\right] / 2$ is shown throughout the RVE. Two volume fractions, $f=15 \%$ and $f=30 \%$, are considered. The CCA bounds on the overall bulk modulus are given for comparison. The PBC always lies within the TBC and DBC. Three main rows correspond to three boundary conditions and three main columns correspond to three different RVEs. For each boundary condition, the center and off-center inclusion, as well as two different volume fractions, are examined.

CCA: composite cylinder assemblage; DBC: displacement boundary condition; PBC: periodic boundary condition; RVE: representative volume element; TBC: traction boundary condition.

Somewhat interestingly, for the bulk modulus, the numerical results indicate that, for random microstructures and for both stiffness ratios, the circular RVE provides a closer overall response to the overall response of the true RVE obtained by the PBC. This is justified by the fact that increasing the number of inclusions within the random microstructure resembles increasing the level of isotropy. Nonetheless, for the periodic microstructure, increasing the number of inclusions does not alter the anisotropy of the material, owing to the uniform distribution of the inclusions. To be more precise, a proper case that could resemble an isotropic material suitable for comparison with analytical bounds is the random microstructure with a large number of inclusions and randomness. Looking at the shear modulus, for the random microstructure with a large number of inclusions, the material response lies within the HSBs for incl. $/$ matr. $=0.1$. However, for incl. $/$ matr. $=10$, the lower HSB is violated and fails to provide a proper bound on the shear modulus. For the Poisson ratio, unlike the shear modulus and the bulk modulus, the TBC provides the stiffest response, whereas the DBC renders the most compliant response.

The studies carried out so far assumed that the inclusion is located at the center of the RVEs. Here, we examine the effect of the inclusion eccentricity by comparing the effective response of the three RVE types due to the canonical boundary conditions for different volume fractions, as well as different stiffness ratios. Figures 13 and 14 provide the deformed shapes of the RVEs, as well as the overall response of the material due to volumetric expansion and simple shear, respectively, for incl. $/$ matr. $=0.1$. The numerical examples correspond to small-strain linear elasticity but deformations are magnified for the 


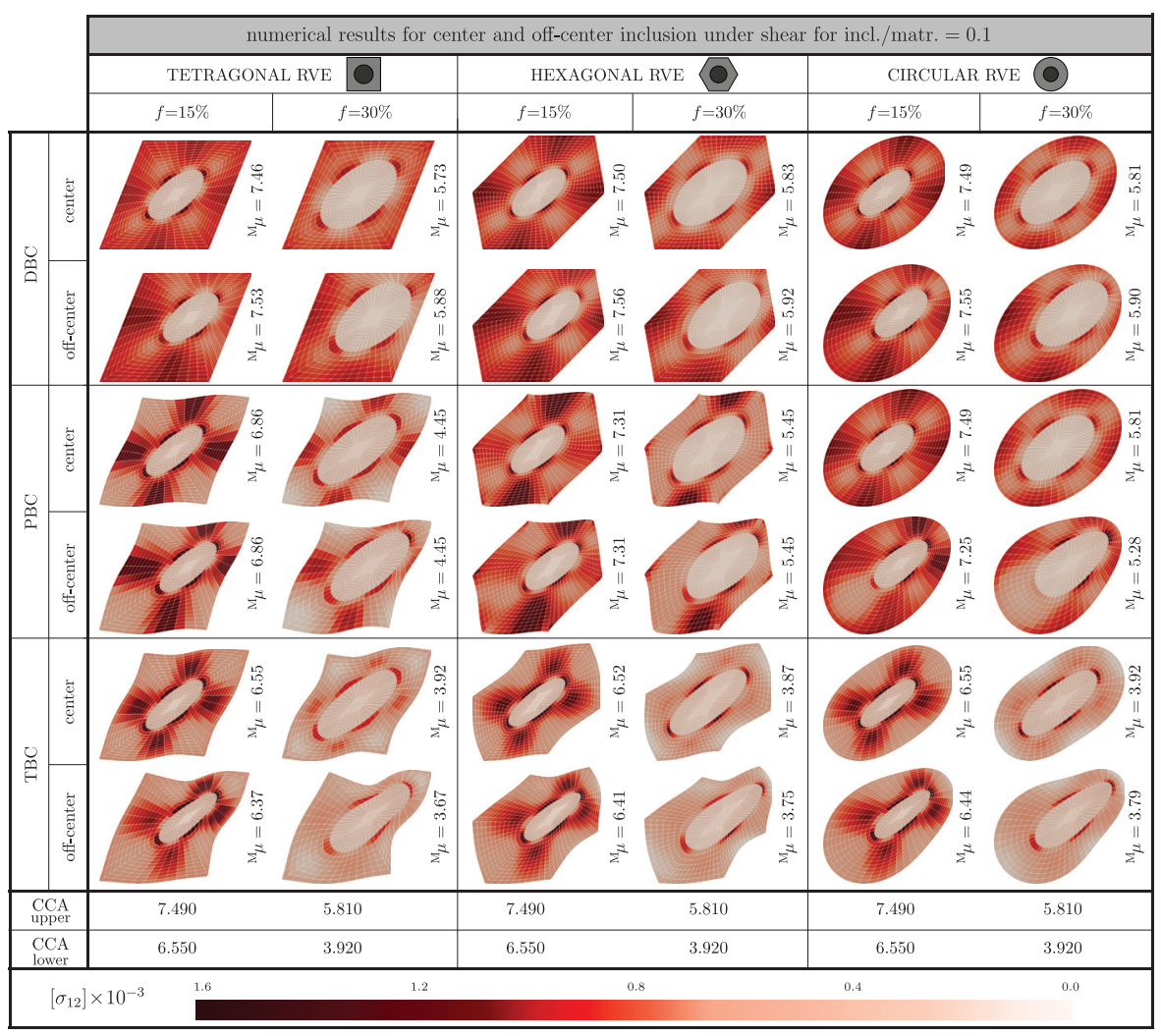

Figure 14. Stress distribution within the RVE under simple shear for both center and off-center inclusions when the inclusion is more compliant than the matrix (incl./matr. $=0.1$ ). The distribution of the stress component $\sigma_{12}$ is shown throughout the RVE. Two volume fractions, $f=15 \%$ and $f=30 \%$, are considered. The CCA bounds on the overall shear modulus are given for comparison. The PBC always lies within the TBC and DBC. Three main rows correspond to three boundary conditions and three main columns correspond to three different RVEs. For each boundary condition, the center and off-center inclusion, as well as two different volume fractions, are examined.

CCA: composite cylinder assemblage; DBC: displacement boundary condition; PBC: periodic boundary condition; RVE: representative volume element; TBC: traction boundary condition.

sake of illustration. Additionally, the distribution of $\left[\sigma_{11}+\sigma_{22}\right] / 2$ for the expansion case and $\sigma_{12}$ for the shear case is displayed throughout each RVE. Each block includes four cases, corresponding to center and off-center inclusions for different volume fractions. The CCA lower and upper bounds are also included to serve as a comparison.

The difference between the results is more distinguishable for $f=30 \%$ than for $f=15 \%$. As expected, the effective bulk modulus for $f=30 \%$ is, in all cases, less than its counterpart for $f=15 \%$. For all the RVEs, when the DBC is prescribed, the off-center inclusion leads to a stiffer response than does the center inclusion. By contrast, if the TBC is imposed, the effective modulus corresponding to the RVE with the off-center inclusion underestimates that with the center inclusion. When the inclusion is at the center of the circular RVE, all the results are identical for a given volume fraction. Somewhat interestingly, when the PBC is employed, the overall response becomes independent of the inclusion position for the tetragonal and hexagonal RVEs. However, for the circular RVE, the material renders a stiffer response for the center inclusion than for the off-center one. Figures 15 and 16 are the counterparts of Figures 13 and 14 , respectively, corresponding to incl./matr. $=10$. The effective moduli, at $f=30 \%$ for all the cases here, are larger than their counterparts at $f=15 \%$. The remainder of the observations in Figures 15 and 16 are similar to those made from Figures 13 and 14; hence, a discussion on them is omitted for the sake of brevity. 


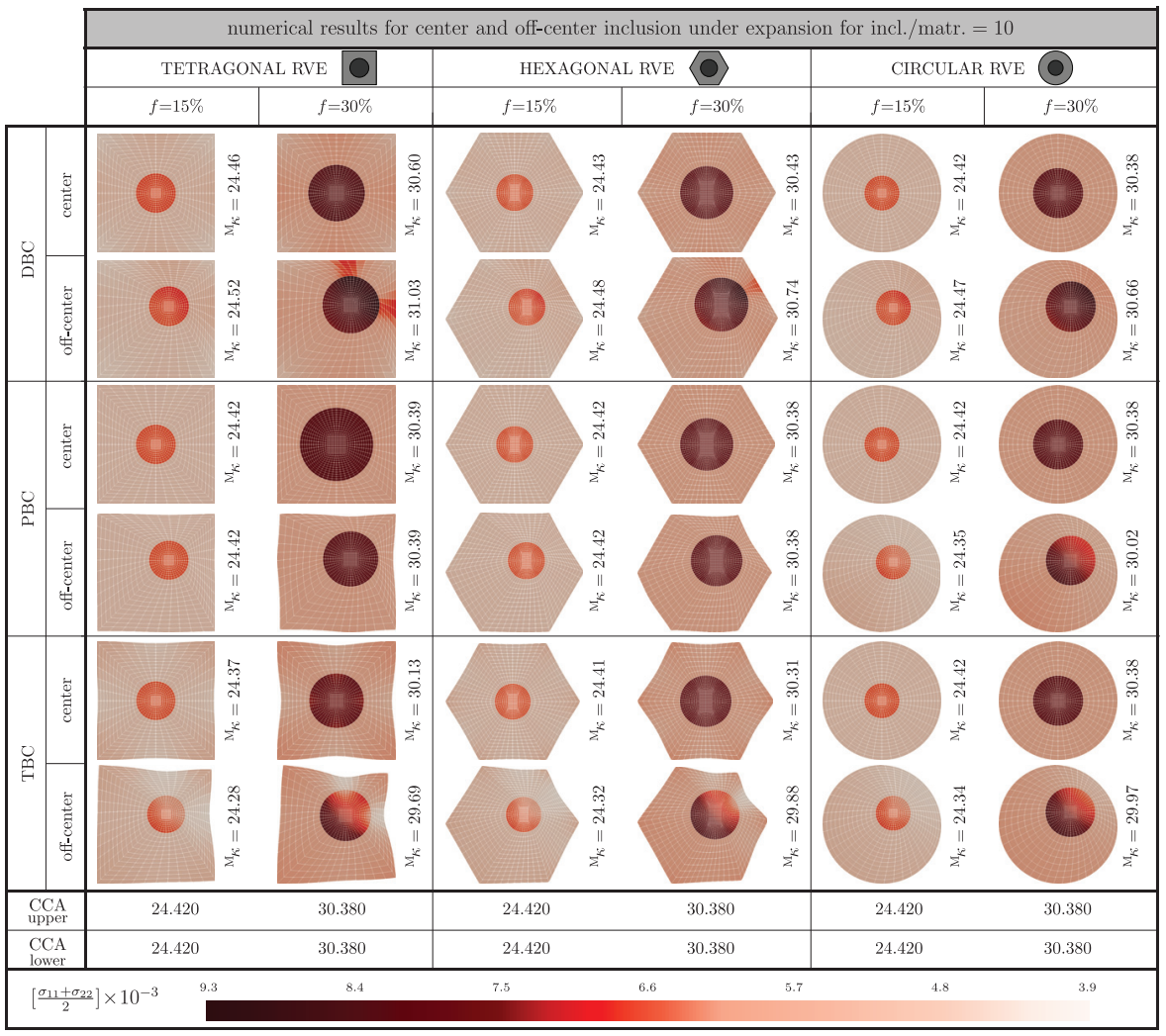

Figure 15. Stress distribution within the RVE under volumetric expansion for both center and off-center inclusions when the inclusion is stiffer than the matrix (incl./matr. $=10$ ). The distribution of the stress component $\left[\sigma_{11}+\sigma_{22}\right] / 2$ is shown throughout the RVE. Two volume fractions $f=15 \%$ and $f=30 \%$ are considered. The CCA bounds on the overall bulk modulus are given for comparison. The PBC always lies within TBC and DBC. Three main rows correspond to three boundary conditions and three main columns correspond to three different RVEs. For each boundary condition, the center and off-center inclusion, as well as two different volume fractions, are examined.

CCA: composite cylinder assemblage; DBC: displacement boundary condition; PBC: periodic boundary condition; RVE: representative volume element; TBC: traction boundary condition.

\section{Extension to finite deformations}

All the previous examples were only valid for small strains. The main objective of this section is to extend the numerical studies presented in the last section to a finite-deformation setting. In doing this, two load cases of volumetric expansion and simple shear are prescribed and the effective material response is computed via proper averaging. The examples carefully analyze the overall material response for various boundary conditions and stiffness ratios. Unlike the case in small-strain linear elasticity, for finite deformations it is not possible to define an effective material parameter, such as bulk modulus or shear modulus. Therefore, in what follows, the apparent macroscopic quantity of interest is the stress itself. For instance, for volumetric expansion, the $x x$-component of the Piola stress and for simple shear, the $x y$-component of the Piola stress are the macroscopic properties of interest.

Figure 17 shows the variation of the macroscopic Piola stress with respect to the volume fraction for various RVEs obtained from different boundary conditions. The first row represents the volumetric expansion case, while the second row corresponds to the simple shear case, both at $20 \%$ deformation. First consider the first row corresponding to the expansion case. As expected, the PBC is bounded with the TBC from below and the DBC from above. For the tetragonal RVE, the results from different boundary conditions mostly deviate from each other as the volume fraction increases. However, as we move toward the circular RVE, the results get closer to each other and eventually coincide. Next consider the second row corresponding to the shear case. For this case, a greater difference between the numerical results is observed, in comparison with the expansion case. The results from the PBC are 


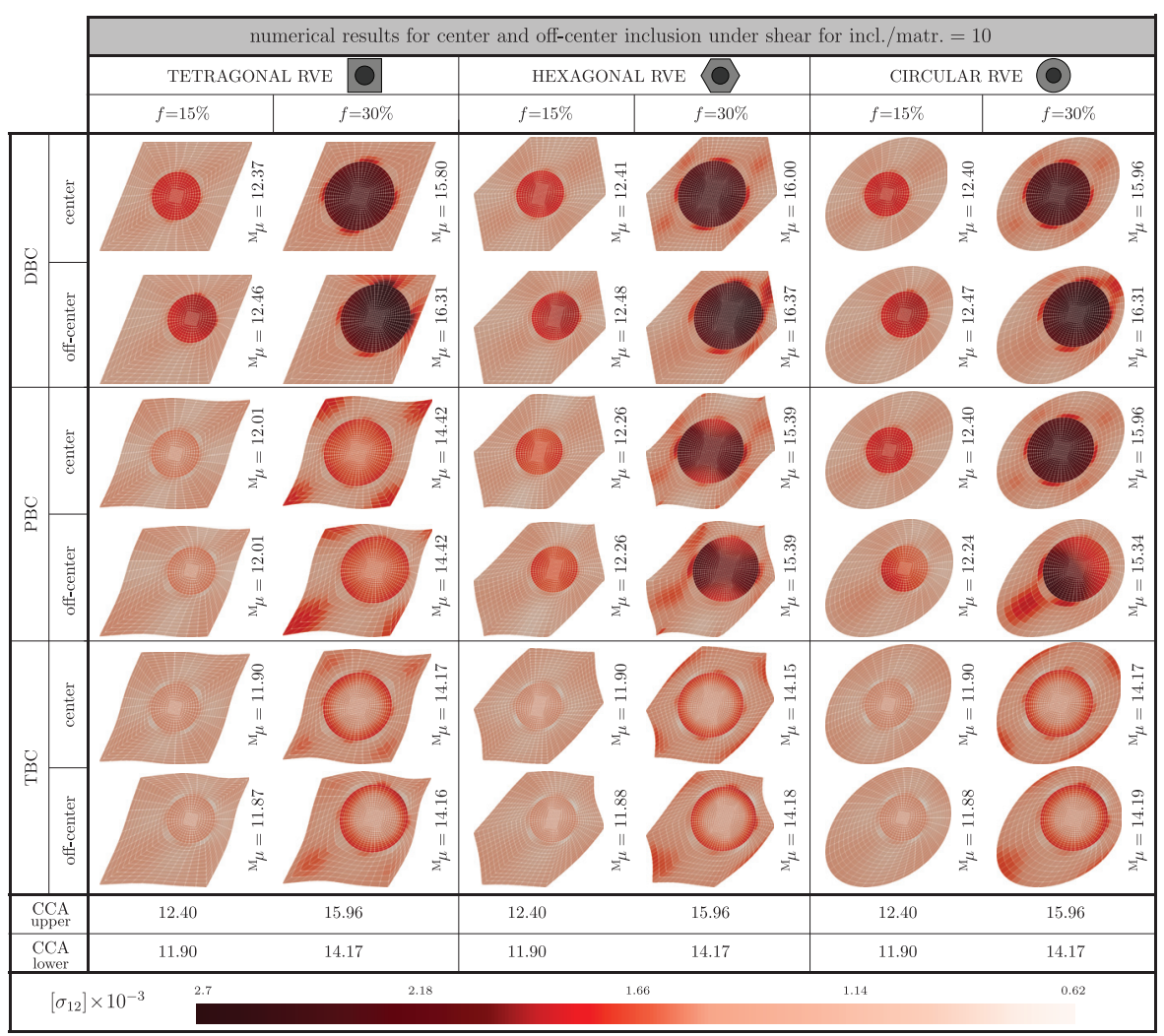

Figure 16. Stress distribution within the RVE under simple shear for both center and off-center inclusions when the inclusion is stiffer than the matrix (incl./matr. = 10). The distribution of the stress component $\sigma_{12}$ is shown throughout the RVE. Two volume fractions, $f=15 \%$ and $f=30 \%$, are considered. The CCA bounds on the overall shear modulus are given for comparison. The PBC always lies within the TBC and DBC. Three main rows correspond to three boundary conditions and three main columns correspond to three different RVEs. For each boundary condition, the center and off-center inclusion, as well as two different volume fractions, are examined.

CCA: composite cylinder assemblage; DBC: displacement boundary condition; PBC: periodic boundary condition; RVE: representative volume element; TBC: traction boundary condition.

closer to the TBC for the tetragonal RVE. For the hexagonal RVE, the PBC renders a closer response than the DBC; for the circular RVE, they result in identical responses. These observations elucidate the sensitivity of the PBC to the RVE type.

Figure 18 depicts the variation of the macroscopic Piola stress with respect to the volume fraction for different boundary conditions. The top row corresponds to the expansion case and the bottom row corresponds to the simple shear case. As mentioned before, the circular RVE is able to result in higher volume fractions than the other RVEs. For the DBC, it is observed that the stress corresponding to the hexagonal RVE is bounded between the tetragonal RVE from above and the circular RVE from below. For the PBC, the same observation is made when expansion is applied. However, when shear is applied, the tetragonal RVE results in the smallest Piola stress, while the hexagonal and circular RVEs render different relative behavior depending on the volume fraction. For the TBC, there is a remarkable coincidence between all the numerical results for both expansion and the shear case. This means that the TBC is indifferent to the type of RVE. This observation highlights the importance of the frequently disregarded TBC in computational homogenization.

\section{Conclusion and outlook}

In summary, we have presented a systematic comparison of the overall behavior of heterogeneous materials as analyzed using both analytical and computational homogenization. It is clearly demonstrated 


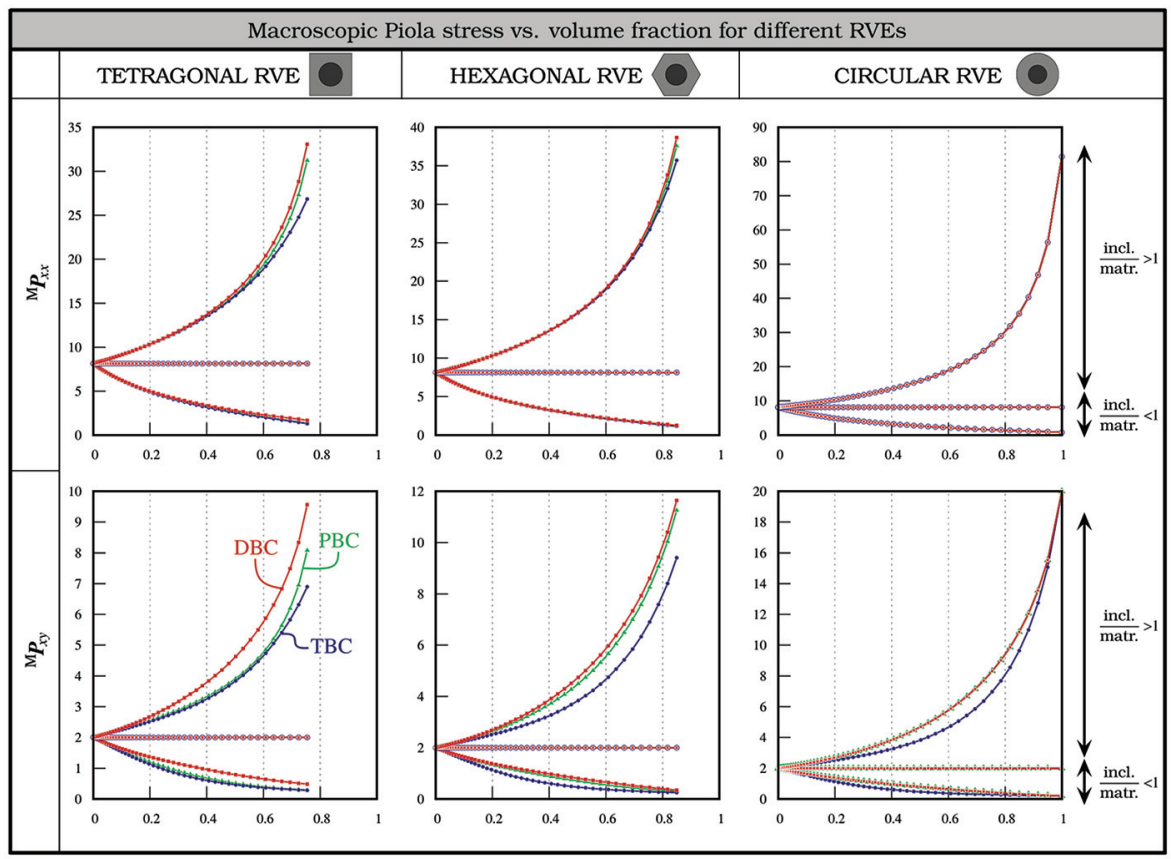

Figure 17. Macroscopic Piola stress with respect to volume fraction $f$ for various types of representative volume element. The top row is associated with the volumetric expansion; thus, the macroscopic stress component of interest is ${ }^{M} P_{x x}$. The bottom row corresponds to the simple shear deformation; thus, the macroscopic stress component of interest is ${ }^{M} P_{x y}$. Each column corresponds to a specific representative volume element. In each graph, different boundary conditions for a specific representative volume element are examined.

DBC: displacement boundary condition; PBC: periodic boundary condition; RVE: representative volume element; TBC: traction boundary condition.

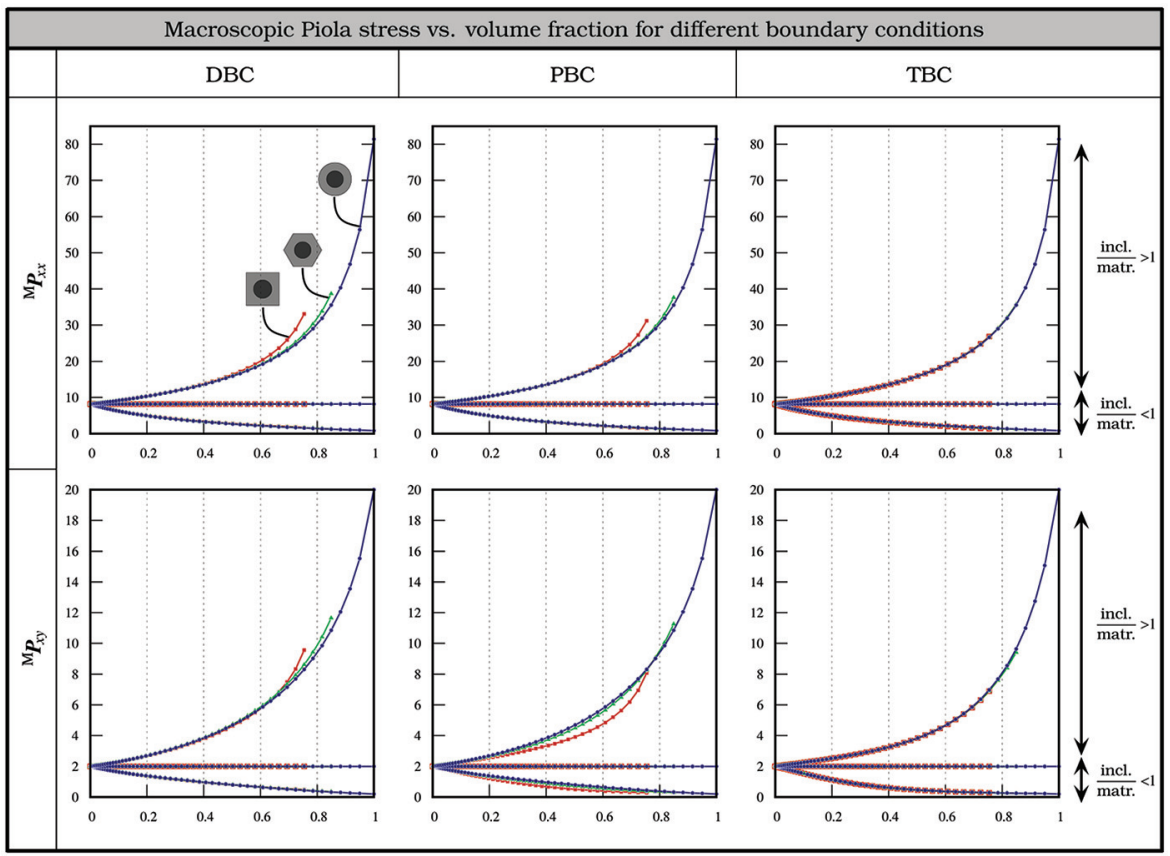

Figure 18. Macroscopic Piola stress with respect to volume fraction $f$ for different boundary conditions. The top row is associated with the volumetric expansion; thus, the macroscopic stress component of interest is ${ }^{M} P_{x x}$. The bottom row corresponds to the simple shear deformation; thus, the macroscopic stress component of interest is ${ }^{M} P_{x y}$. Each column corresponds to a specific boundary condition. In each graph, different representative volume element types for a specific boundary condition are examined. DBC: displacement boundary condition; PBC: periodic boundary condition; TBC: traction boundary condition. 
that, in contrast to the Voigt and Reuss bounds, for some cases the HSBs do not provide reliable bounds on the shear modulus. Three different material properties, i.e. bulk modulus, shear modulus, and Poisson ratio were comprehensively studied for various RVEs, boundary conditions, and microstructures. Our findings show that of all the simplified RVE types, the circular RVE serves as the most suitable RVE to predict overall isotropic material behavior, owing to its intrinsic ability to capture isotropy. The influence of inclusion eccentricity on the effective modulus of the material is examined. For both tetragonal and hexagonal packings, the numerical results based on the PBC are indifferent with respect to the inclusion position in the RVE. The numerical results are then extended to the finitedeformation setting; it is observed that the TBC shows less sensitivity with respect to RVE type. This conclusion is particularly interesting, since the TBC is often disregarded in computational homogenization. Our next immediate extension of this contribution deals with a complementary study, accounting for size effects. Further extensions of this work include three-dimensional analysis, as well as the inclusion of more complex analytical estimates.

\section{Acknowledgements}

Ali Javili would like to thank Christian Linder for inspiring discussions regarding the circular RVE.

\section{Funding}

The author(s) disclosed receipt of the following financial support for the research, authorship, and/or publication of this article: This work was supported by the Cluster of Excellence "Engineering of Advanced Materials" at the University of ErlangenNuremberg, funded by the DFG within the framework of its "Excellence Initiative".

\section{ORCID iDs}

George Chatzigeorgiou (iD https://orcid.org/0000-0002-7213-2980

Ali Javili (iD https://orcid.org/0000-0001-7965-7088

\section{References}

[1] Broughton, J, Abraham, F, Bernstein, N, et al. Concurrent coupling of length scales: Methodology and application. Phys Rev B 1999; 60: 2391-2403.

[2] Oden, JT, Vemaganti, K, and Moes, N. Hierarchical modeling of heterogeneous solids. Comput Methods Appl Mech Eng 1999; 172: 3-25.

[3] Ghosh, S, Bai, J, and Raghavan, P. Concurrent multi-level model for damage evolution in microstructurally debonding composites. Mech Mater 2007; 39: 241-266.

[4] Khoei, AR, Jahanbakhshi, F, and Aramoon, A. A concurrent multi-scale technique in modeling heterogeneous FCC nano-crystalline structures. Mech Mater 2015; 83: 40-65.

[5] Suquet, PM. Elements of homogenization theory for inelastic solid mechanics. In: Sanchez-Palencia, E, and Zaoui, A (eds.) Homogenization techniques for composite media (Lecture Notes in Physics, vol. 272). Berlin: Springer, 1987, 194-278.

[6] Moulinec, H, and Suquet, P. A numerical method for computing the overall response of nonlinear composites with complex microstructure. Comput Methods Appl Mech Eng 1998; 157: 69-94.

[7] Ponte Castañeda, P, and Suquet, P. Nonlinear composites. Adv Appl Mech 1997; 34: 171-302.

[8] van der Sluis, O, Vosbeek, P, Schreurs, P, et al. Homogenization of heterogeneous polymers. Int J Solids Struct 1999; 36: 3193-3214.

[9] Zohdi, TI, and Wriggers, P. Computational micro-macro material testing. Arch Comput Methods Eng 2001; 8: 131-228.

[10] Kouznetsova, V, Geers, MGD, and Brekelmans, WAM. Multi-scale constitutive modelling of heterogeneous materials with a gradient-enhanced computational homogenization scheme. Int J Numer Methods Eng 2002; 54: 1235-1260.

[11] Lloberas-Valls, O, Rixen, DJ, Simone, A, et al. On micro-to-macro connections in domain decomposition multiscale methods. Comput Methods Appl Mech Eng 2012; 225: 177-196.

[12] Li, X, Liu, Q, and Zhang, J. A micro-macro homogenization approach for discrete particle assembly-Cosserat continuum modeling of granular materials. Int J Solids Struct 2010; 47: 291-303.

[13] Willoughby, N, Parnell, WJ, Hazel, AL, et al. Homogenization methods to approximate the effective response of random fibre-reinforced composites. Int J Solids Struct 2012; 49: 1421-1433.

[14] Jiang, WG, Zhong, RZ, Qin, QH, et al. Homogenized finite element analysis on effective elastoplastic mechanical behaviors of composite with imperfect interfaces. Int J Mol Sci 2014; 15: 23389-23407. 
[15] Raina, A, and Linder, C. A homogenization approach for nonwoven materials based on fiber undulations and reorientation. J Mech Phys Solids 2014; 65: 12-34.

[16] Hill, R. Elastic properties of reinforced solids: Some theoretical principles. J Mech Phys Solids 1963; 11: 357-372.

[17] Hill, R. On constitutive macro-variables for heterogeneous solids at finite strain. Proc R Soc London, Ser A 1972; 326: 131-147.

[18] Ogden, R. On the overall moduli of non-linear elastic composite materials. J Mech Phys Solids 1974; $22: 541-553$.

[19] Mandel, J. Contribution théorique à l'étude de l'écrouissage et des lois de l'écoulement plastique. In: Görtler, H (ed.) Applied mechanics. Berlin, Springer, 1966, 502-509.

[20] Voigt, W. Ueber die Beziehung zwischen den beiden Elasticitätsconstanten isotroper Körper. Ann Phys 1889; 274: 573-587.

[21] Reuss, A. Berechnung der Fließgrenze von Mischkristallen auf Grund der Plastizitätsbedingung für Einkristalle. ZAMM 1929; 9: 49-58.

[22] Eshelby, JD. The determination of the elastic field of an ellipsoidal inclusion, and related problems. Proc R Soc London, Ser A 1957; 241: 376-396.

[23] Hashin, Z, and Shtrikman, S. A variational approach to the theory of the elastic behaviour of multiphase materials. $J$ Mech Phys Solids 1963; 11: 127-140.

[24] Hashin, Z, and Rosen, B. The elastic moduli of fiber-reinforced materials. J Appl Mech 1964; 31: $223-232$.

[25] Hill, R. Theory of mechanical properties of fibre-strengthened materials: I. Elastic behaviour. J Mech Phys Solids 1964; 12: 199-212.

[26] Hill, R. A self-consistent mechanics of composite materials. J Mech Phys Solids 1965; 13: 213-222.

[27] Walpole, LJ. On the overall elastic moduli of composite materials. J Mech Phys Solids 1969; 17: $235-251$.

[28] Mori, T, and Tanaka, K. Average stress in matrix and average elastic energy of materials with misfitting inclusions. Acta Metall 1973; 21: 571-574.

[29] Willis, JR. Bounds and self-consistent estimates for the overall properties of anisotropic composites. J Mech Phys Solids 1977; 25: 185-202.

[30] Nemat-Nasser, S, and Iwakuma, T. Elastic-plastic composites at finite strains. Int J Solids Struct 1985; 21: 55-65.

[31] Willis, JR. On methods for bounding the overall properties of nonlinear composites. J Mech Phys Solids 1991; 39: 73-86.

[32] Ponte Castañeda, P. The effective mechanical properties of nonlinear isotropic composites. J Mech Phys Solids 1991; 39: 45-71.

[33] Torquato, S. Random heterogeneous media: Microstructure and improved bounds on effective properties. Appl Mech Rev 1991; 44: 37-76.

[34] Ponte Castañeda, P, DeBotton, G, and Li, G. Effective properties of nonlinear inhomogeneous dielectrics. Phys Rev B 1992; 46: 4387-4394.

[35] Nemat-Nasser, S, Yu, N, and Hori, M. Bounds and estimates of overall moduli of composites with periodic microstructure. Mech Mater 1993; 15: 163-181.

[36] Balendran, B, and Nemat-Nasser, S. Bounds on elastic moduli of composites. J Mech Phys Solids 1995; 43 : $1825-1853$.

[37] Kochmann, DM, and Milton, GW. Rigorous bounds on the effective moduli of composites and inhomogeneous bodies with negative-stiffness phases. $J$ Mech Phys Solids 2014; 71: 46-63.

[38] Nemat-nasser, S, and Hori, M. Universal bounds for overall properties of linear and nonlinear heterogeneous solids. $J$ Eng Mater Technol 2015; 117: 412-432.

[39] Klusemann, B, Bohm, H, and Svendsen, B. Homogenization methods for multi-phase elastic composites with nonelliptical reinforcements: Comparisons and benchmarks. Eur J Mech A Solids 2012; 34: 21-37.

[40] Brinson, LC, and Knauss, WG. Finite element analysis of multiphase viscoelastic solids. J Appl Mech 1992; 59: $730-737$.

[41] Brinson, LC, and Lin, WS. Comparison of micromechanics methods for effective properties of multiphase viscoelastic composites. Compos Struct 1998; 41: 353-367.

[42] Terada, K, and Kikuchi, N. A class of general algorithms for multi-scale analyses of heterogeneous media. Comput Methods Appl Mech Eng 2001; 190: 5427-5464.

[43] Miehe, C, and Koch, A. Computational micro-to-macro transitions of discretized microstructures undergoing small strains. Arch Appl Mech 2002; 72: 300-317.

[44] Feyel, F. A multilevel finite element method $\left(\mathrm{FE}^{2}\right)$ to describe the response of highly non-linear structures using generalized continua. Comput Methods Appl Mech Eng 2003; 192: 3233-3244.

[45] Moës, N, Cloirec, M, Cartraud, P, et al. A computational approach to handle complex microstructure geometries. Comput Methods Appl Mech Eng 2003; 192: 3163-3177.

[46] Özdemir, I, Brekelmans, WAM, and Geers, MGD. Computational homogenization for heat conduction in heterogeneous solids. Int J Numer Methods Eng 2008; 73: 185-204.

[47] Fritzen, F, and Leuschner, M. Reduced basis hybrid computational homogenization based on a mixed incremental formulation. Comput Methods Appl Mech Eng 2013; 260: 143-154. 
[48] Javili, A, Chatzigeorgiou, G, and Steinmann, P. Computational homogenization in magneto-mechanics. Int J Solids Struct 2013; 50: 4197-4216.

[49] Kochmann, DM, and Venturini, GN. Homogenized mechanical properties of auxetic composite materials in finite-strain elasticity. Smart Mater Struct 2013; 22: 084004.

[50] Biswas, R, and Poh, LH. A micromorphic computational homogenization framework for heterogeneous materials. $J$ Mech Phys Solids 2017; 102: 187-208.

[51] Larsson, F, Runesson, K, and Su, F. Variationally consistent computational homogenization of transient heat flow. Int J Numer Methods Eng 2010; 81: 1659-1686.

[52] Feyel, F, and Chaboche, JL. FE ${ }^{2}$ multiscale approach for modelling the elastoviscoplastic behaviour of long fibre $\mathrm{SiC} / \mathrm{Ti}$ composite materials. Comput Methods Appl Mech Eng 2000; 183: 309-330.

[53] Fritzen, F, and Leuschner, M. Nonlinear reduced order homogenization of materials including cohesive interfaces. Comput Mech 2015; 56: 131-151.

[54] Tikarrouchine, E, Praud, F, Chatzigeorgiou, G, et al. Three-dimensional FE ${ }^{2}$ method for the simulation of non-linear, rate-dependent response of composite structures. Compos Struct 2018; 193: 165-179.

[55] Chatzigeorgiou, G, Charalambakis, N, Chemisky, Y, et al. Thermomechanical behavior of dissipative composite materials. London: ISTE Press-Elsevier, 2018.

[56] Saeb, S, Steinmann, P, and Javili, A. Aspects of computational homogenization at finite deformations: A unifying review from Reuss' to Voigt's bound. Appl Mech Rev 2016; 68: 050801.

[57] Geers, MGD, Kouznetsova, V, and Brekelmans, WAM. Multi-scale computational homogenization: Trends and challenges. J Comput Appl Math 2010; 234: 2175-2182.

[58] Charalambakis, N, Chatzigeorgiou, G, Chemisky, Y, et al. Mathematical homogenization of inelastic dissipative materials: A survey and recent progress. Continuum Mechanics and Thermodynamics 2018; 30: 1-51.

[59] Yadegari, S, Turteltaub, S, and Suiker, AS. Generalized grain cluster method for multiscale response of multiphase materials. Comput Mech 2015; 56: 193-219.

[60] Matsui, K, Terada, K, and Yuge, K. Two-scale finite element analysis of heterogeneous solids with periodic microstructures. Comput Struct 2004; 82: 593-606.

[61] Michel, JC, Moulinec, H, and Suquet, P. Effective properties of composite materials with periodic microstructure: a computational approach. Comput Methods Appl Mech Eng 1999; 172: 109-143.

[62] Yvonnet, J, Monteiro, E, and He, QC. Computational homogenization method and reduced database model for hyperelastic heterogeneous structures. Int J Multiscale Comput Eng 2013; 11: 201-225.

[63] Le, B, Yvonnet, J, and He, Q. Computational homogenization of nonlinear elastic materials using neural networks. Int J Numer Methods Eng 2015; 104: 1061-1084.

[64] Suquet, PM. Local and global aspects in the mathematical theory of plasticity. Plast Today 1985; 1985: $279-309$.

[65] Pierard, O, Friebel, C, and Doghri, I. Mean-field homogenization of multi-phase thermo-elastic composites: A general framework and its validation. Compos Sci Technol 2004; 64: 1587-1603.

[66] Mercer, BS, Mandadapu, KK, and Papadopoulos, P. Novel formulations of microscopic boundary-value problems in continuous multiscale finite element methods. Comput Methods Appl Mech Eng 2015; 286: 268-292.

[67] Yuan, Z, and Fish, J. Toward realization of computational homogenization in practice. Int J Numer Methods Eng 2008; 73: 361-380.

[68] Nguyen, VD, Béchet, E, Geuzaine, C, et al. Imposing periodic boundary condition on arbitrary meshes by polynomial interpolation. Comput Mater Sci 2012; 55: 390-406.

[69] Pecullan, S, Gibiansky, LV, and Torquato, S. Scale effects on the elastic behavior of periodic and hierarchical twodimensional composites. J Mech Phys Solids 1999; 47: 1509-1542.

[70] Hazanov, S, and Huet, C. Order relationships for boundary conditions effect in heterogeneous bodies smaller than the representative volume. J Mech Phys Solids 1994; 42: 1995-2011.

[71] Hazanov, S, and Amieur, M. On overall properties of elastic heterogeneous bodies smaller than the representative volume. Int J Eng Sci 1995; 33: 1289-1301.

[72] Pahr, DH, and Zysset, PK. Influence of boundary conditions on computed apparent elastic properties of cancellous bone. Biomech Model Mechanobiol 2008; 7: 463-476.

[73] Terada, K, Hori, M, Kyoya, T, et al. Simulation of the multi-scale convergence in computational homogenization approaches. Int J Solids Struct 2000; 37: 2285-2311.

[74] Yuan, X, and Tomita, Y. Effective properties of Cosserat composite with periodic microstructure. Mech Res Commun 2001; 28: 265-270.

[75] Jiang, M, Alzebdeh, K, Jasiuk, I, et al. Scale and boundary conditions effects in elastic properties of random composites. Acta Mech 2001; 148: 63-78.

[76] Ostoja-Starzewski, M. Material spatial randomness: From statistical to representative volume element. Probab Eng Mech 2006; 21: 112-132.

[77] Drago, A, and Pindera, MJ. Micro-macromechanical analysis of heterogeneous materials: Macroscopically homogeneous vs periodic microstructures. Compos Sci Technol 2007; 67: 1243-1263. 
[78] Saroukhani, S, Vafadari, R, Andersson, R, et al. On statistical strain and stress energy bounds from homogenization and virtual testing. Eur $J$ Mech A Solids 2015; 51: 77-95.

[79] Nguyen, VD, Wu, L, and Noels, L. Unified treatment of microscopic boundary conditions and efficient algorithms for estimating tangent operators of the homogenized behavior in the computational homogenization method. Comput Mech 2017; 59: 483-505.

[80] Gitman, IM, Askes, H, and Aifantis, EC. The representative volume size in static and dynamic micro-macro transitions. Int J Fract 2005; 135: 3-9.

[81] Khisaeva, ZF, and Ostoja-Starzewski, M. On the size of RVE in finite elasticity of random composites. $J$ Elast 2006; 85: 153-173.

[82] Temizer, I, and Zohdi, TI. A numerical method for homogenization in non-linear elasticity. Comput Mech 2007; 40: 281-298.

[83] Temizer, I, Wu, T, and Wriggers, P. On the optimality of the window method in computational homogenization. Int $J$ Eng Sci 2013; 64: 66-73.

[84] Thomas, M, Boyard, N, Perez, L, et al. Representative volume element of anisotropic unidirectional carbon-epoxy composite with high-fibre volume fraction. Compos Sci Technol 2008; 68: 3184-3192.

[85] Dirrenberger, J, Forest, S, and Jeulin, D. Towards gigantic RVE sizes for 3D stochastic fibrous networks. Int J Solids Struct 2014; 51: 359-376.

[86] Schröder, J, Balzani, D, and Brands, D. Approximation of random microstructures by periodic statistically similar representative volume elements based on lineal-path functions. Arch Appl Mech 2011; 81: 975-997.

[87] Balzani, D, Scheunemann, L, Brands, D, et al. Construction of two- and three-dimensional statistically similar RVEs for coupled micro-macro simulations. Comput Mech 2014; 54: 1269-1284.

[88] Glüge, R, Weber, M, and Bertram, A. Comparison of spherical and cubical statistical volume elements with respect to convergence, anisotropy, and localization behavior. Comput Mater Sci 2012; 63: 91-104.

[89] Miehe, C. Computational micro-to-macro transitions for discretized micro-structures of heterogeneous materials at finite strains based on the minimization of averaged incremental energy. Comput Methods Appl Mech Eng 2003; 192: 559-591.

[90] Kanouté, P, Boso, DP, Chaboche, JL, et al. Multiscale methods for composites: A review. Arch Comput Methods Eng 2009; 16: 31-75.

[91] Matous, K, Geers, MG, Kouznetsova, VG, et al. A review of predictive nonlinear theories for multiscale modeling of heterogeneous materials. J Comput Phys 2017; 330: 192-220.

[92] Kouznetsova, V, Brekelmans, WAM, and Baaijens, FPT. An approach to micro-macro modeling of heterogeneous materials. Comput Mech 2001; 27: 37-48.

[93] Ladevèze, P, Loiseau, O, and Dureisseix, D. A micro-macro and parallel computational strategy for highly heterogeneous structures. Int J Numer Methods Eng 2001; 52: 121-138.

[94] Miehe, C, and Dettmar, J. A framework for micro-macro transitions in periodic particle aggregates of granular materials. Comput Methods Appl Mech Eng 2004; 193: 225-256.

[95] Stolz, C. On micro-macro transition in non-linear mechanics. Materials (Basel) 2010; 3: 296-317.

[96] Liu, Q, Liu, X, Li, X, et al. Micro-macro homogenization of granular materials based on the average-field theory of Cosserat continuum. Adv Powder Technol 2014; 25: 436-449.

[97] Chatzigeorgiou, G, Chemisky, Y, and Meraghni, F. Computational micro to macro transitions for shape memory alloy composites using periodic homogenization. Smart Mater Struct 2015; 24: 035009.

[98] Chatzigeorgiou, G, Meraghni, F, and Javili, A. Generalized interfacial energy and size effects in composites. J Mech Phys Solids 2017; 106: 257-282.

[99] Larsson, F, Runesson, K, Saroukhani, S, et al. Computational homogenization based on a weak format of microperiodicity for RVE-problems. Comput Methods Appl Mech Eng 2011; 200: 11-26.

[100] Javili, A, Saeb, S, and Steinmann, P. Aspects of implementing constant traction boundary conditions in computational homogenization via semi-Dirichlet boundary conditions. Comput Mech 2017; 59: 21-35.

[101] Hashin, Z. On elastic behaviour of fibre reinforced materials of arbitrary phase transverse geometry. $J$ Mech Phys Solids 1965; 13: 119-134.

[102] Walpole, LJ. On bounds for the overall elastic moduli of inhomogeneous systems-I. J Mech Phys Solids 1966; 14: 151-162.

[103] Weng, GJ. Explicit evaluation of Willis' bounds with ellipsoidal inclusions. Int J Eng Sci 1992; 30: 83-92.

[104] Hashin, Z. Analysis of composite materials-A survey. J Appl Mech 1983; 50: 481-505.

[105] Savvas, D, Stefanou, G, Papadrakakis, M, et al. Homogenization of random heterogeneous media with inclusions of arbitrary shape modeled by XFEM. Comput Mech 2014; 54: 1221-1235.

[106] Ghosh, S, Lee, K, and Moorthy, S. Multiple scale analysis of heterogeneous elastic structures using homogenization theory and Voronoi cell finite element method. Int J Solids Struct 1995; 32: 27-62. 


\section{Appendix}

\section{Notation}

$\begin{array}{ll}\mathbb{A} & \text { Piola tangent } \\ \mathcal{B} & \text { Body of the domain } \\ \boldsymbol{F} & \text { Deformation gradient } \\ f & \text { Volume fraction } \\ \boldsymbol{N} & \text { Unit normal vector } \\ \boldsymbol{P} & \text { Piola stress } \\ \boldsymbol{t} & \text { Traction vector } \\ \boldsymbol{\kappa} & \text { Bulk modulus } \\ \lambda & \text { First Lamé parameter } \\ \mu & \text { Second Lamé parameter } \\ \nu & \text { Poisson ratio } \\ \boldsymbol{\varphi} & \text { Nonlinear deformation map } \\ \psi & \text { Free energy density } \\ \partial \mathcal{B} & \text { Boundary of the domain }\end{array}$

\section{Subscripts and superscripts}

$\begin{array}{ll}\{\bullet\}_{\mathrm{D}} & \text { Dirichlet part of }\{\bullet\} \\ \left.\mathrm{M}_{\{}\right\} & \text {Macroscopic }\{\bullet\} \\ \{\bullet\}_{\mathrm{N}} & \text { Neumann part of }\{\bullet\} \\ \{\bullet\}^{\mathrm{P}} & \text { Prescribed }\{\bullet\} \\ \{\bullet\}_{t} & \{\bullet\} \text { at spatial configuration } \\ \{\bullet\}_{0} & \{\bullet\} \text { at material configuration }\end{array}$

\section{Abbreviations}

$\begin{array}{ll}\text { CCA } & \text { Composite cylinder assemblage } \\ \text { DBC } & \text { Displacement boundary condition } \\ \text { HSB } & \text { Hashin-Shtrikman bounds } \\ \text { PBC } & \text { Periodic boundary condition } \\ \text { RVE } & \text { Representative volume element } \\ \text { TBC } & \text { Traction boundary condition }\end{array}$

